\title{
Transcriptome wide analyses reveal a sustained cellular stress response in the gill tissue of Trematomus bernacchii after acclimation to multiple stressors
}

Troy J. Huth ${ }^{2}$ and Sean P. Place ${ }^{1 *}$

\begin{abstract}
Background: As global climate change progresses, the Southern Ocean surrounding Antarctica is poised to undergo potentially rapid and substantial changes in temperature and $p \mathrm{CO}_{2}$. To survive in this challenging environment, the highly cold adapted endemic fauna of these waters must demonstrate sufficient plasticity to accommodate these changing conditions or face inexorable decline. Previous studies of notothenioids have focused upon the short-term response to heat stress; and more recently the longer-term physiological response to the combined stress of increasing temperatures and $\mathrm{pCO}_{2}$. This inquiry explores the transcriptomic response of Trematomus bernacchii to increased temperatures and $p \mathrm{CO}_{2}$ at 7, 28 and 56 days, in an attempt to discern the innate plasticity of $T$. bernacchii available to cope with a changing Southern Ocean.
\end{abstract}

Results: Differential gene expression analysis supported previous research in that $T$. bernacchii exhibits no inducible heat shock response to stress conditions. However, T. bernacchii did demonstrate a strong stress response to the multi-stressor condition in the form of metabolic shifts, DNA damage repair, immune system processes, and activation of apoptotic pathways combined with negative regulation of cell proliferation. This response declined in magnitude over time, but aspects of this response remained detectable throughout the acclimation period.

Conclusions: When exposed to the multi-stressor condition, T. bernacchii demonstrates a cellular stress response that persists for a minimum of 7 days before returning to near basal levels of expression at longer acclimation times. However, subtle changes in expression persist in fish acclimated for 56 days that may significantly affect the fitness $T$. bernacchii over time.

Keywords: Ocean acidification, Thermal stress, RNA-seq, Notothenioid, Gene expression

\section{Background}

Isolation of the Antarctic continental shelf by the Polar Front has arguably produced the coldest, most oceanographically stable environment on the planet. However, this long-term oceanographic stability may have resulted in the evolution of an ecosystem filled with endemic fauna that are poorly poised to deal with rapid climate variation $[1,2]$. In the face of global climate change, marine organisms are perceived to have but three

\footnotetext{
*Correspondence: places@sonoma.edu

'Department of Biology, Sonoma State University, Rohnert Park, CA 94928, USA

Full list of author information is available at the end of the article
}

options: they can migrate to more favorable environments, alter their biology through physiological plasticity, or evolve in response to the altered environment [3-7]. Given the unique environment afforded by the Southern Ocean, it is highly unlikely that population migration is a viable option for its endemic biota. While evolutionary responses may benefit the species in some longer time frame, they may be outpaced by environmental change. Thus, for extant communities in the Antarctic, use of physiologically plastic responses may be the only available method of persisting under nearterm future environmental variations. 
The Southern Ocean is dominated by an endemic suborder of perciform fishes, the Notothenioidei [8]. The effects of increased temperature on the physiology of Antarctic fish have been well documented providing some important insight into the plasticity of a number of Antarctic fish species to a single stress [9-16]. However, we have very little information regarding what impact interacting and synergistic stressors will have on the physiological tolerances of these unique fish. This is problematic for identifying physiological tipping-points for polar species, as previous measurements of their capacity to respond may be overly conservative since they do not account for non-additive effects of the joint action of multiple stressors. This point has been highlighted by recent studies that assessed the physiological response of temperate and eurythermal organisms to the combined stress of ocean acidification and elevated temperature. Results reported from these studies suggest significant trade-offs in performance and stress tolerance may exist when two or more environmental conditions are varied [17-19]. An important outcome of these studies is an increased understanding among physiological ecologists that relevant assessment of species vulnerabilities will require consideration of multiple environmental variables [20, 21]. Equally important is the realization that it is no longer sufficient to focus on a single cellular or molecular response, but rather the consideration of multiple co-regulated processes is critical [20,22].

Genomics-based approaches hold the promise of greatly facilitating our understanding of physiological plasticity in these endemic fishes, especially when considering the impact dynamic environments have on multiple physiological pathways [23-26]. With modern genomic techniques, we can now ask: to what extent are conserved patterns of gene expression absent in the Antarctic fishes and how does this affect their ability to adjust to major changes in their environment? To this end, we have used RNA-seq analyses to profile the genomic response of an endemic Antarctic fish to predicted levels of ocean acidification and global increases in mean sea surface temperature (SST). In this investigation of multiple stressors related to climate change, our goal was to assess the molecular response of Trematomus bernacchii to conditions consistent with scenarios laid out by the IPCC with respect to anthropogenic increases in atmospheric $\mathrm{CO}_{2}$ [6] while providing direct comparison to previous studies looking at thermal stress in this organism.

\section{Results and Discussion}

\section{Reference transcriptome}

Following assembly, the transcriptomic library initially consisted of 421,044 unigenes (unique gene products including all isoforms) and 537,064 transcripts with a median transcript length of $444 \mathrm{bp}$, mean transcript length of $1011 \mathrm{bp}$ and N50 of $2160 \mathrm{bp}$. After removing transcripts with expression levels below 0.001 FPKM (fragments per kilobase million) and clustering at $100 \%$ identity; 314,638 transcripts and 246,333 unigenes remained. Transcript level annotation yielded 98,451 BLAST hits $\left(1 \times 10 \mathrm{e}^{-6}\right.$ cutoff value) and 34,096 GO (gene ontology) annotations.

\section{Sequencing read quality control and mapping}

Sequencing yielded an average of 27,141,939 reads (s.d. $= \pm 2,544,475 ; \quad \min =22,080,886 ; \quad \max =30,834,415)$ per sample. Trimmomatic [27] quality processing retained an average of $94.28 \%$ (s.d. $= \pm 0.14 \%$; $\min =93.99 \%$; $\max =94.58 \%)$ of the input reads; resulting in samples containing an average of 25,591,193 reads (s.d. $= \pm 2,413,885$; $\min =20,760,581 ; \max =29,083,777)$. Bowtie2 [28] mapping achieved an average of $90.90 \%$ (s.d. $= \pm 5.76 \%$; $\mathrm{min}=$ $89.52 \% ; \max =91.72 \%)$ of the trimmed sequencing reads mapped to the reference transcriptome, which corresponded to an average number of mapped reads per sample of $23,261,884$ (s.d. $= \pm 2,206,537 ; \min =18,817,366$; $\max =26,562,213)$.

\section{Transcriptome-wide differential gene expression analysis}

Differential gene expression analysis using edgeR [29] yielded a total of 4880 differentially expressed genes across all three time points (FDR $\leq 0.05)$. A sample similarity comparison demonstrated that the $7 \mathrm{~d}$ multi-stressor treatment resulted in considerable differential gene expression when compared to the $7 \mathrm{~d}$ control treatment (Fig. 1). Furthermore, the $7 \mathrm{~d}$ multi-stressor individuals clustered as an outgroup in the cluster dendrogram of all treatments and time-points, demonstrating the consistent and distinct effect of this treatment-time combination on overall gene expression as compared to all others (Fig. 1). The $28 \mathrm{~d}$ and $56 \mathrm{~d}$ multi-stressor treatments also segregated from their respective control treatments indicating differential expression compared to the control, although to a lesser extent than the $7 \mathrm{~d}$ multi-stressor treatment (Fig. 1).

Direct comparisons of the control and multi-stressor treatment at each time-point demonstrates 2528 differentially expressed genes within the $7 \mathrm{~d}$ multi-stressor treatment; 209 differentially expressed genes within the 28d multi-stressor treatment; and 419 differentially expressed genes within the $56 \mathrm{~d}$ multi-stressor treatment. Of the 2528 differentially expressed genes of the $7 d$ multi-stressor treatment, 1642 were up-regulated and 886 were down-regulated (Fig. 2). Of the 209 differentially expressed genes in the $28 \mathrm{~d}$ multi-stressor treatment 123 were up-regulated with 86 down-regulated (Fig. 2). Lastly, the 56d multi-stressor treatment demonstrated 187 up-regulated genes and 232 down-regulated genes out of the 419 total differentially expressed (Fig. 2). 


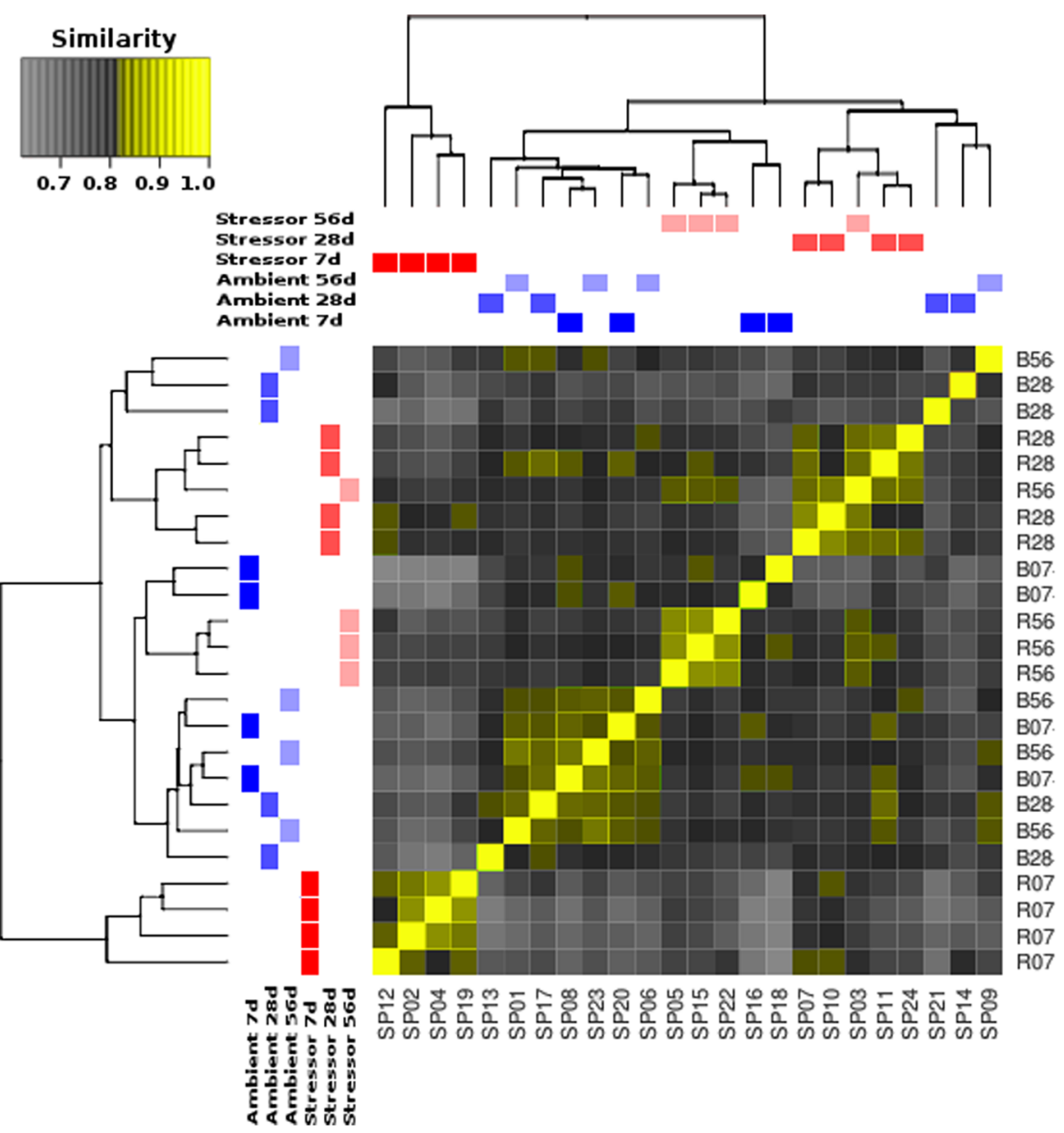

Fig. 1 Transcriptome wide sample similarity matrix and cluster dendrogram: the sample similarity matrix represents the cumulative similarity of each individual at each time-point as a reflection of transcriptome wide gene expression. Transcriptome wide expression is represented by all gene products that demonstrated an FDR $\leq 0.05$ during the differential gene expression analysis. Portions of the matrix shown in yellow demonstrate a high degree of similarity in the transcriptomic expression profiles between the two samples, with a value of 1 indicating the samples are identical; whereas those shown in grey demonstrate a lower degree of similarity, with a value of 0.65 demonstrating the most dissimilar expression profiles between two samples. As the samples are extracted from the same tissue and species, the degree of similarity in this case is a minimum of 0.65 . The cluster dendrogram also groups samples based upon similar expression profiles, with those samples grouped most closely demonstrating more analogous transcriptomic expression responses

These metrics indicate a robust initial response to the $7 \mathrm{~d}$ multi-stressor treatment, which tapers off considerably in fish acclimated to the multi-stressor treatment for $28 \mathrm{~d}$ and $56 \mathrm{~d}$ (Fig. 2). A slight increase in the number of differentially expressed genes was observed between the $28 \mathrm{~d}$ and $56 \mathrm{~d}$ time-points which may provide insight into the long-term acclimation tactics in these fish (Fig. 2).

\section{Gene ontology over-representation analysis}

Fisher's Exact Tests for gene ontology term overrepresentation further supported a robust initial transcriptome-wide response in gill tissues that diminishes over time, with the $7 \mathrm{~d}$ stressor treatment exhibiting the most differentially expressed and over-represented gene ontology terms.

For fish within the $7 \mathrm{~d}$ multi-stress acclimation group, GO terms within the molecular function subclasses associated with differentially up-regulated genes indicate these fish experienced large-scale cellular remodeling (Fig. 3, Additional file 1: Table S1). Among the upregulated genes in the $7 \mathrm{~d}$ multi-stressor treatment we found that GO terms associated with nucleic acid binding, transcription factor activity, helicase activity, and double-stranded RNA binding activity were overrepresented; indicating a significant change in transcription activation and RNA processing. Furthermore, the increased expression of GTPase activity, GTP binding, 


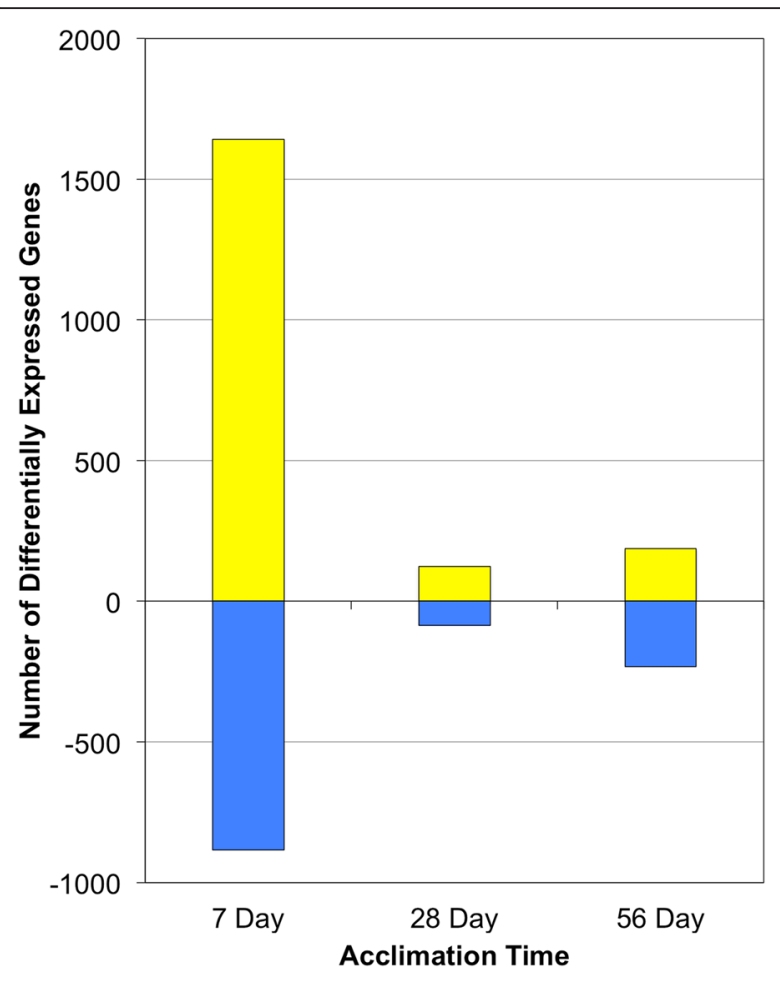

Fig. 2 Number of differentially expressed genes: the bar graph depicts the total number of differentially expressed genes observed between the multi-stressor treatment and control fish at each time point. Yellow bars (positive values) represent the total number of DE genes up-regulated after $7,28, \& 56$ days of acclimation while blue bars (negative values) represent the total number of DE genes down-regulated. In all, differential gene expression analysis using edgeR [29] yielded a total of 4880 differentially expressed genes across all three time points (FDR $\leq 0.05$ ). Fish from the $7 \mathrm{~d}$ multi-stressor treatment displayed 1642 up-regulated and 886 down-regulated. Fish from the $28 \mathrm{~d}$ multi-stressor treatment displayed 123 up-regulated and 86 down-regulated. Fish from the 56d multi-stressor treatment displayed 187 up-regulated and 232 down-regulated

and tRNA ligase activity suggests a significant upregulation of a diverse number of cellular functions such as trafficking across the nuclear membrane and protein biosynthesis in the $7 \mathrm{~d}$ multi-stressor-acclimated group. Lastly, there also appears to be a considerable amount of protein recycling occurring in fish acclimated to the multi-stress treatment for 7 days as evidence by the significant increase in the expression of 23 genes associated with peptidase activity. This spike in protein degradation coincides with a marked increase in oxidative damaged in the same fish tissue [30]. It has been previously demonstrated that transcriptional regulation, RNA processing, protein biosynthesis and proteolysis are highly active gene ontology groups when exposing $T$. bernacchii to short term heat stress $\left(4{ }^{\circ} \mathrm{C}\right.$ for $\left.4 \mathrm{~h}\right)$ [31]; our findings indicate that this initial cellular stress response continues well into the first 7 days. It was further observed that peptidase activity remained up-regulated in fish acclimated to the multi-stressor treatment for $28 \mathrm{~d}$, before being down-regulated in the $56 \mathrm{~d}$ stressor treatment specimens.

Over-representation analysis of GO terms in the biological processes category identified a significant number of genes associated with a sustained response to cellular damage in the gill cells of fish acclimated to the $7 d$ multi-stressor treatment (Fig. 4, Additional file 1: Table S1). The top 4 over-represented categories included immune system response (23 genes), response to stress (19), cell death (11), and protein ubiquitination (5). Whereas the biological processes highly over-represented in the downregulated genes in the gill tissues of the same fish include signal transduction (54 genes), embryo or morphological development (11), and cell proliferation (6). The overrepresentation of any biological processes is largely absent in fish acclimated to the multi-stressor treatment for $28 \mathrm{~d}$, while we observed a slight increase in the positive regulation of the cell cycle (8) and DNA metabolic processes (5) in the $56 \mathrm{~d}$ multi-stressor treatment. Taken together, the analysis of molecular and biological functions suggest $T$. bernacchii maintains a sustained response to cellular stress in its gills for at least 7 days and returns to a homeostatic state by 28 days of acclimation. Specimens acclimated to the multi-stressor treatment may experience a second adjustment in transcriptional activity that may indicate a potentially prolonged acclimation response at the $56 \mathrm{~d}$ time-point.

Organisms experiencing environmental stress can often display two conserved responses, a rapid, transient response known as the cellular stress response (CSR) and a more permanent response termed the cellular homeostasis response (CHR) [32]. It is likely expression profiles observed in the $7 \mathrm{~d}$ and then the $28 \mathrm{~d}$ or $56 \mathrm{~d}$ acclimated fish highlights the transition from the CSR to the CHR, and are representative of both the immediate and long-term adjustments necessary to cope with these environmental conditions. Therefore, a more detailed analysis of the biological processes found to be overrepresented was conducted for cell death and immune system process; in addition to a number of more specific $\mathrm{GO}$ terms for biological processes potentially involved in the acclimation to multiple stressors including: carbohydrate metabolic processes, lipid metabolic processes, cell proliferation, cell death, response to stress, and homeostatic processes. These sub-categories are further investigated below in an attempt to identify specific gene products involved in the initial $7 \mathrm{~d}$ time-point response, and those that may indicate a capacity for long term acclimation at the $28 \mathrm{~d}$ and $56 \mathrm{~d}$ time-points (Table 1).

To highlight the molecular response of these fish to acute and chronic stress, we have separated our discussion into two primary sections consisting of the short- 


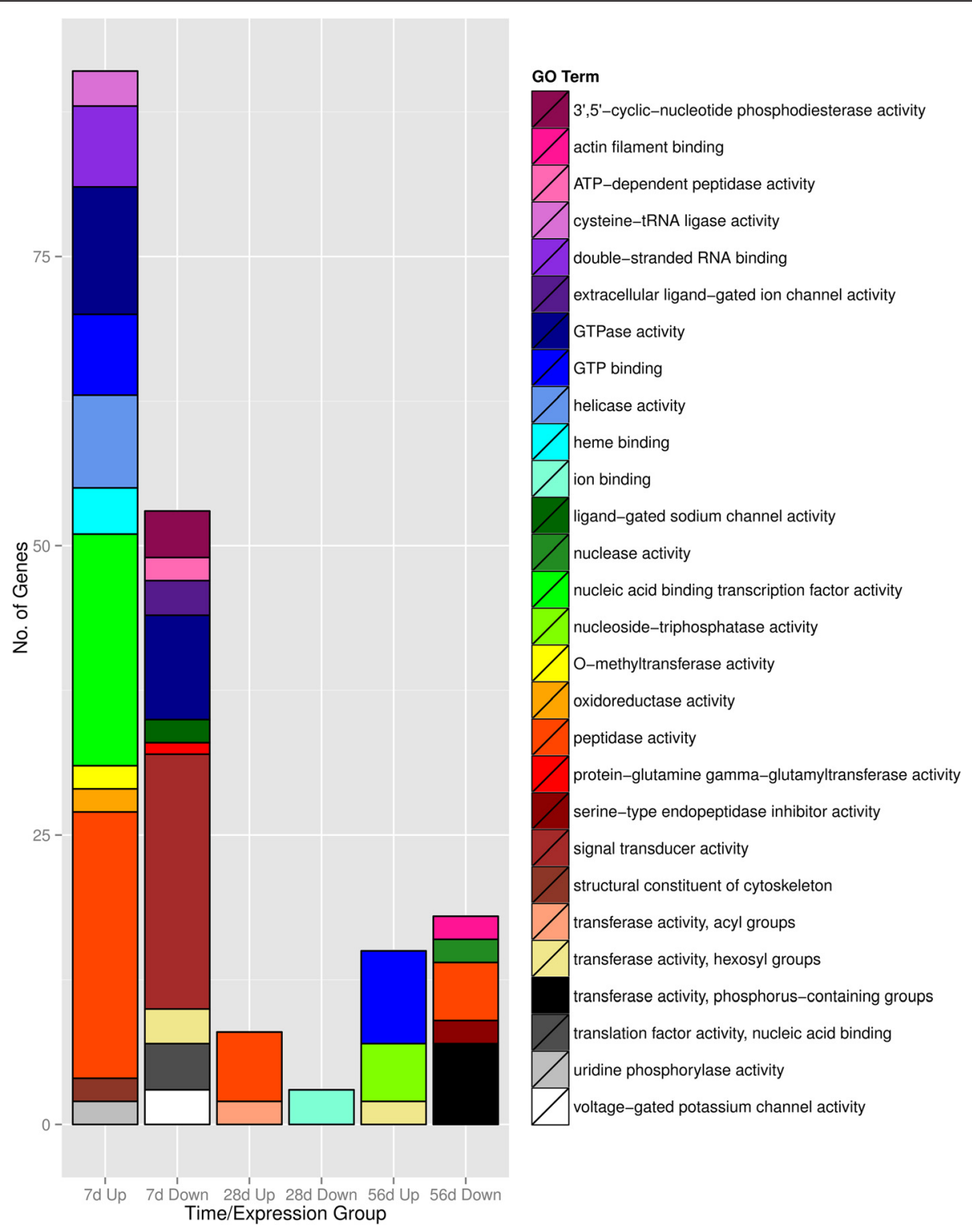

Fig. 3 Over-representation analysis of gene ontology level 2 category molecular function: shown are gene ontology terms within the broad molecular function category (GO:0003674) that were significantly over-represented in the stressor treatments compared to the reference transcriptome as determined by a Fisher's exact test ( $p<0.01$, categories containing only one gene are not included for readability). Subgroups of up- and down-regulated gene cohorts were created for each stressor time-point $(7 \mathrm{~d}, 28 \mathrm{~d}$ and $56 \mathrm{~d})$ and compared to the $\mathrm{GO}$ term distribution of the reference transcriptome. The number in each category represents the total number of genes within that over-expressed GO term category $(b l a n k=0)$

term responses $(7 \mathrm{~d})$ and the longer-term responses $(28$ and 56d).

\section{Pathway specific responses $(7 \mathrm{~d}$ multi-stressor treatment)}

Carbohydrate and lipid metabolism

Analysis of significant changes in mRNA expression levels for genes associated with the GO category for carbohydrate metabolism (Fig. 5, Additional file 2: Table S2) demonstrated a nearly equal number of genes that were up- and down-regulated at $7 \mathrm{~d}$ in fish acclimated to the multi-stressor treatment relative to the control treatment (30 up-regulated, 31 down-regulated). Of the up-regulated genes in this category, nearly half (12) were associated with hydrolysis of carbohydrates while 13 genes displaying significant down-regulation were associated with carbohydrate synthesis. The same general trend was observed in lipid metabolism, with 20 genes up-regulated and 22 genes down-regulated in fish acclimated to the multi-stressor treatment for $7 \mathrm{~d}$. Of the genes found to be up-regulated at the $7 \mathrm{~d}$ time-point, a large number of genes were associated with small molecule metabolic processes 


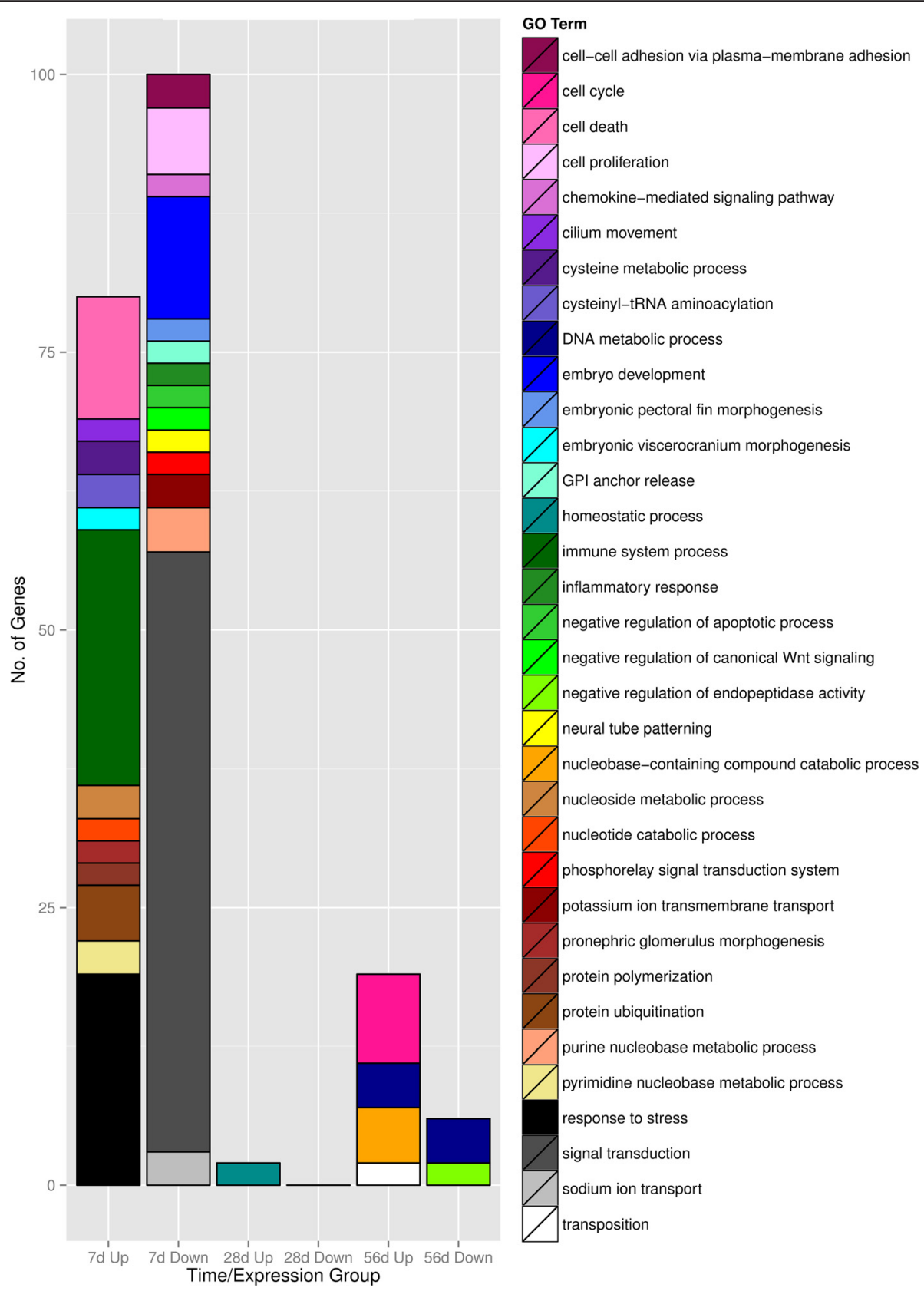

Fig. 4 Over-representation analysis of the gene ontology level 2 category biological process: gene ontology terms within the broad biological process category (GO:0008150) that were significantly over-represented in the stressor treatments compared to the reference transcriptome as determined by a Fisher's exact test ( $p<0.01$, categories containing only one gene are not included for readability). Subgroups of up- and downregulated gene cohorts were created for each stressor time-point (7d, 28d and 56d) and compared to the $\mathrm{GO}$ term distribution of the reference transcriptome. The number in each category represents the total number of genes within that over-expressed GO term category (blank $=0$ )

(11), most notably genes involved in phospholipid biosynthesis and membrane maintenance such as SERINC5, PLCXD1, PLCXD3, and EPT1. Among the downregulated genes, 16 were associated with lipid biosynthesis or catabolism (Fig. 5, Additional file 3: Table S3).

Previous heat stress studies in Notothenioids have demonstrated an up-regulation of genes associated with both carbohydrate and lipid metabolism, albeit it on a much shorter time scale of $4 \mathrm{~h}$ [31]. An increased glycolytic capacity is paralleled by a significant increase in resting metabolic rates when specimens are acclimated to stressful conditions for 7 days [33]. The rapid increase in capacity and oxygen consumption likely fuels the massive cellular reorganization captured in 
Table 1 A summary of major gene ontology groups demonstrating differential gene expression at the 7d, 28d, and 56d time-points of the multi-stressor condition

\begin{tabular}{|c|c|c|c|c|c|c|c|c|c|c|}
\hline \multirow[t]{2}{*}{ Gene ontology category } & \multirow{2}{*}{$\begin{array}{l}\text { Total genes } \\
\text { found in the } \\
\text { transcriptome }\end{array}$} & \multicolumn{3}{|c|}{ 7d differentially expressed } & \multicolumn{3}{|c|}{ 28d differentially expressed } & \multicolumn{3}{|c|}{ 56d differentially expressed } \\
\hline & & Total & Up & Down & Total & Up & Down & Total & Up & Down \\
\hline Carbohydrate metabolic process & 480 & 61 & 30 & 31 & 2 & 2 & 0 & 9 & 8 & 1 \\
\hline Cell death & 171 & 32 & 20 & 12 & 3 & 1 & 2 & 4 & 1 & 3 \\
\hline Cell proliferation & 155 & 19 & 4 & 15 & 0 & 0 & 0 & 1 & 1 & 0 \\
\hline Homeostatic process & 194 & 20 & 18 & 2 & 2 & 2 & 0 & 9 & 5 & 4 \\
\hline Immune system process & 246 & 53 & 39 & 14 & 7 & 7 & 0 & 9 & 5 & 4 \\
\hline Lipid metabolic process & 409 & 42 & 20 & 22 & 4 & 4 & 0 & 8 & 6 & 2 \\
\hline Response to stress & 420 & 62 & 48 & 14 & 3 & 3 & 0 & 12 & 7 & 5 \\
\hline
\end{tabular}

The first column includes the total number of genes found within the transcriptome associated with each GO term, followed by the differential expression data for each time-point of the multi-stressor treatments as compared to the control treatment (sub-divided into total, up- and down-regulated) for each of the GO term categories carbohydrate metabolic process, cell death, cell proliferation, homeostatic process, immune system process, lipid metabolic process, and response to stress. The confidence threshold to determine differential expression was a FDR $\leq 0.05$

the expression profiles of fish acclimated to the multistressor treatment for 7 days. We have more recent metabolic rate data that provides further support for this hypothesis. The metabolic rates of these fish were measured over the $56 \mathrm{~d}$ acclimation period, and similar to the 2013 study [33], these fish showed a dramatic increase in resting metabolic rate (RMR) within the first $7 \mathrm{~d}$ of acclimation to the multi-stressor treatment, however, RMR rates in these fish returned to basal levels by $56 \mathrm{~d}$ [34].

Additionally, notothenioids are thought to rely primarily on lipids to fuel glycolytic metabolism under nonstressed conditions [12, 35]; however, the significant down-regulation of lipid mobilization and catabolism pathways observed after 7 days of acclimation to stressful conditions suggests $T$. bernacchii has shifted away from its reliance on lipids as a primary energy source for ATP generation. This is further reinforced by the simultaneous up-regulation of carbohydrate hydrolysis and down-regulation of carbohydrate synthesis pathways in addition to a nearly 2 -fold increase in lactate dehydrogenase (LDH, 1.8-fold). Similar shifts in apparent glycolytic substrate preference have been noted for a number of other Antarctic species via changes in enzyme activity for LDH, citrate synthase (CS), cytochrome-c oxidase (cyt-c) and hydroxyacyl-CoA dehydrogenase (HOAD). In specimens of a closely related notothenioid, Pagothenia borchgrevinki, acclimated to elevated temperature, both LDH and cyt-c activity were found to be elevated [36]. A distantly related species, the eelpout Pachycara brachycephalum, exhibited increased cyt-c activity coupled with decreased CS activity at elevated temperatures [37]. Lastly, Jayasundara et al. reported a similar increase in LDH activity coupled with decreased CS activities in T. bernacchii specimens acclimated to $+4.5^{\circ} \mathrm{C}[38]$.

\section{Cellular death and proliferation}

In addition to the metabolic changes that were seen in the gill tissue of $7 d$ multi-stressor acclimated fish, we also observed a significant change in the regulation of cell survival with the activation of several genes associated with apoptosis (Fig. 6, Additional file 4: Table S4). Gill tissue isolated from these fish demonstrated a significant up-regulation of a number of caspases (1.7 to 3.0-fold); and multiple suppressors of cytokine signaling (4.5 to 7.4-fold). Interestingly, a down regulation of angiopoietin-related proteins ( -2.3 to 4.2 -fold,) and endothelial pas domain-containing proteins $(-2.2$ to -2.3 -fold), which can activate the cell's hypoxia response, are also observed at $7 \mathrm{~d}$.

As to cell proliferation, fish in the $7 \mathrm{~d}$ multi-stressor treatment demonstrated a strong trend of down-regulation overall (4 up-regulated, 20 down-regulated) (Fig. 6, Additional file 5: Table S5) that suggests cell cycle arrest in response to stress extends beyond the initial timeframe proposed by Sleadd and colleagues [39]. Specifically, we observed a strong down-regulation in a number of growth factors including insulin growth factors $(-7.7$ to -13.0 -fold); fibroblast growth factors $(-3.6$ to -12.1 fold); myostatin (-4.6-fold); bone morphogenic protein ( -3.4 to -3.6 -fold), and platelet-derived growth factor (-3.0-fold), among others.

\section{Response to stress}

Our results support previous studies, which found a lack of inducible heat shock response in T. bernacchii [26, 40, 41]. Despite the considerable transcriptome-wide changes in expression demonstrated above; of the 64 heat shock and heat shock-related genes expressed in the reference transcriptome, only 7 were found to be differentially regulated (FDR $\leq 0.05$ ) in fish exposed to the 7d multi-stressor treatment (Fig. 7, Additional file 6: 


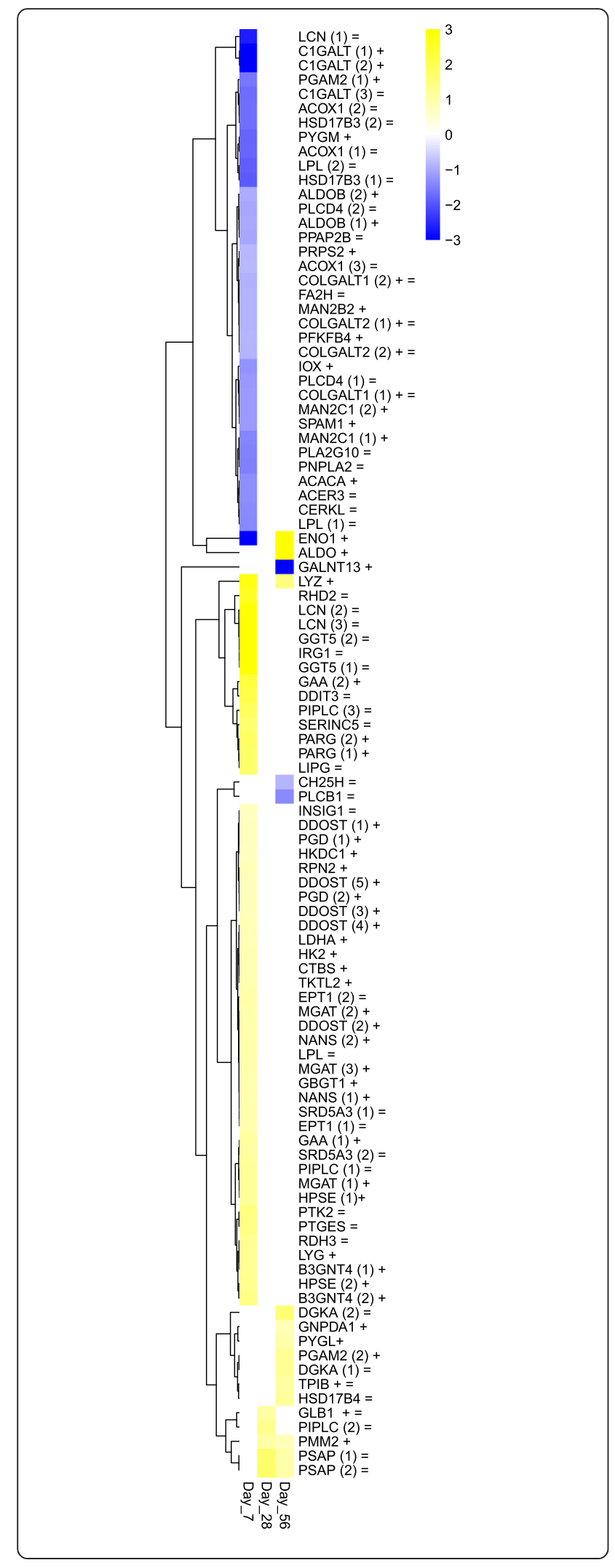

Fig. 5 Heatmap-metabolic processes: this heatmap demonstrates the change in expression patterns of metabolic genes that displayed differential expression in at least one acclimation time point $(7 \mathrm{~d}$, $28 \mathrm{~d}$, or $56 \mathrm{~d}$ ) when fish acclimated to the multi-stressor treatment $(n=4)$ were compared to fish in the control treatments $(n=4)$. Genes associated with the gene ontology term carbohydrate metabolic process (GO:0005975) are represented by (+) and lipid metabolic process (GO:0006629) are represented by (=) . Fold change is $\log _{2}$ adjusted with genes showing significant up-regulation depicted in yellow and genes showing a significant down-regulation in blue (FDR $\leq 0.05$ ). Genes whose level of expression did not differ significantly between control and multi-stressor treatments are shown in white. The number of gene variants identified in the annotated transcriptome that were associated with the same gene name are indicated in parentheses next the gene symbol

Table S6). Overall, of the three HSP families typically induced in a teleost heat shock response [42], there is almost no response for the HSP90, HSP70 and small HSP families. Among those heat shock proteins that did show significant changes in regulation, HSP40 demonstrated the clearest up-regulation with both HSP40 genes found to be up-regulated (2.0 and 1.9-fold) in the $7 \mathrm{~d}$ multistressor treatment group. A similar trend was observed in $P$. borchgrevinki acclimated to ${ }^{+} 4{ }^{\circ} \mathrm{C}$ for 4 days. In this study, Bilyk and Cheng report HSP47 as the only HSP transcript displaying significant up-regulation in this closely related species [25].

While none of the inducible HSP isoforms were differentially regulated, there did appear to be a number of endoplasmic reticulum (ER) specialized molecular chaperones that did show moderate increases in the $7 d$ multi-stressor treatment. For instance, of the over 20 HSP70-related genes annotated in our reference library, only 2 transcripts ( $h s p 70-12 b, 2.3$-fold and hsp70-14, 1.9-fold) demonstrated significant up-regulation (2.3-fold and 1.9-fold, respectively). Similarly, GRP94, a member of the Hsp90 family that plays a role in assembly of secreted proteins and is localized in the ER, was upregulated $\sim 2.5$-fold in the $7 \mathrm{~d}$ fish.

In opposition to the lack of a robust HSR, T. bernacchii does display a strong response to DNA damage. The gill tissue from fish acclimated to the multi-stressor treatment for $7 \mathrm{~d}$ displayed up-regulation of a number of genes associated with activation of DNA damage response pathways (Fig. 7, Additional file 7: Table S7). Among the genes up-regulated are several members of the GADD45 family of proteins which mediate the activation of the p38/JNK pathway, resulting in cell cycle arrest, DNA repair, cell senescence, and apoptosis [43]. Furthermore, two key genes directly involved in DNA repair also displayed significant increases. Proliferating cell nuclear antigen (PCNA), which has a stimulatory effect on the $33^{\prime}-5$ ' exonuclease activity of DNA polymerase and RAD2, which is a structure-specific $5^{\prime}$-flap 


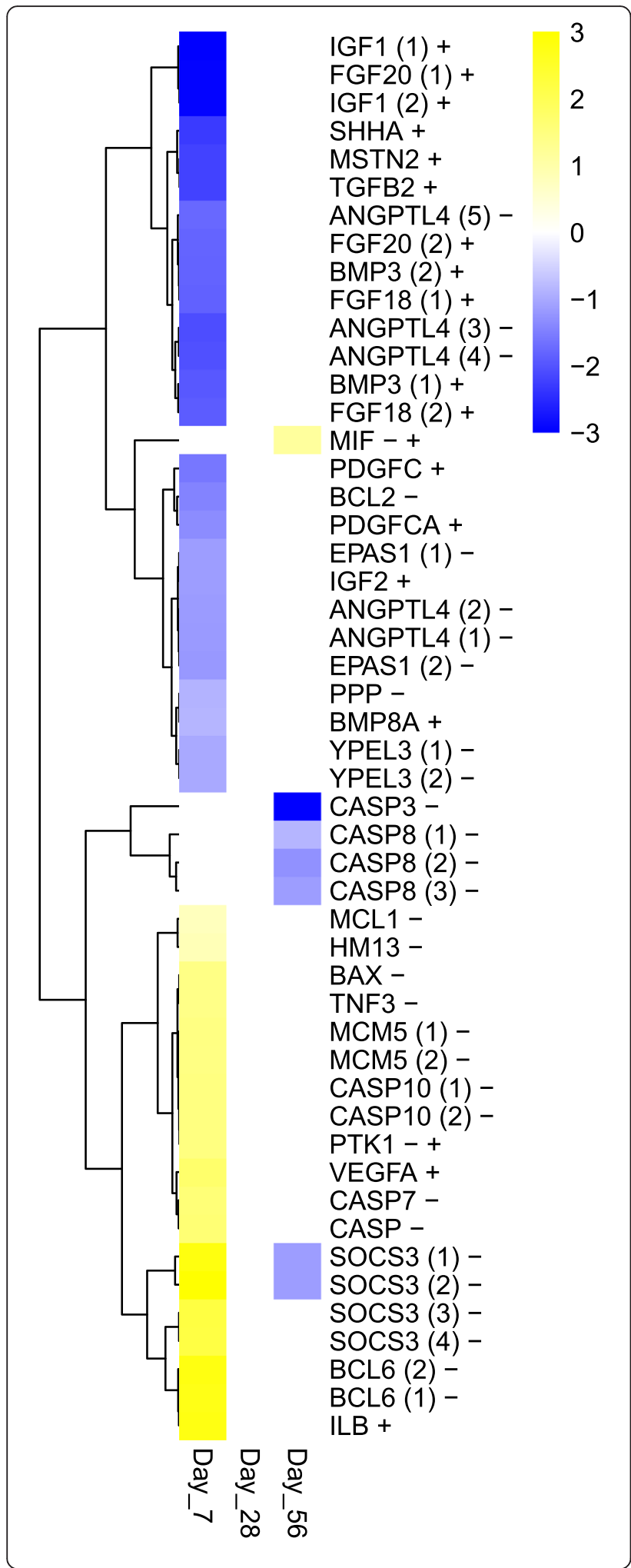

Fig. 6 Heatmap apoptosis and cell proliferation: this heatmap demonstrates the change in expression patterns of genes involved in cell proliferation or programmed cell death that displayed differential expression in at least one acclimation time point $(7 \mathrm{~d}$, $28 \mathrm{~d}$, or 56d) when fish acclimated to the multi-stressor treatment $(n=4)$ were compared to fish in the control treatments $(n=4)$. Genes associated with the gene ontology term cell death (GO:0008219) are represented by (-) and cell proliferation (GO:0008283) are represented by $(+)$. Fold change is $\log _{2}$ adjusted with genes showing significant up-regulation depicted in yellow and genes showing a significant down-regulation in blue (FDR $\leq 0.05)$. Genes whose level of expression did not differ significantly between control and multi-stressor treatments are shown in white. The number of gene variants identified in the annotated transcriptome that were associated with the same gene name are indicated in parentheses next the gene symbol

endonuclease, were both up-regulated over 2 -fold in the $7 \mathrm{~d}$ multi-stressor acclimated fish.

In one of the few studies that has looked at cell proliferation and DNA damage in Antarctic fish under conditions of cellular stress, Sleadd et al. found a significant increase in PCNA protein concentrations in T. bernacchii after being held at ${ }^{+} 4{ }^{\circ} \mathrm{C}$ for $72 \mathrm{~h}$ [39]. Unlike our findings at the transcript level, Sleadd et al. found that PCNA protein levels had returned to control values by $168 \mathrm{~h}$ (7d). In our multi-stressor study, we found PCNA transcript levels were still significantly elevated in the gill tissue of $T$. bernacchii after a $7 \mathrm{~d}$ acclimation that included a ${ }^{+} 4{ }^{\circ} \mathrm{C}$ thermal stress. The elevation in this DNA repair machinery tracks the molecular signatures of cellcycle arrest described above. It is possible the additional stressor (elevated $p \mathrm{CO}_{2}$ ) extended the response of PCNA and cell cycle arrest in our study; however, given the differences in tissues observed between the two studies (gill vs liver) and the molecule considered (mRNA vs protein) it is difficult to draw more concrete conclusions.

\section{Immune system processes}

An aggressive immune response is a common characteristic of teleosts when stressed from any number of cellular perturbations [44] and this appeared to hold true for T. bernacchii. Genes associated with immune system processes demonstrated a general trend of up-regulation in the $7 \mathrm{~d}$ multi-stressor treatment (39 up-regulated, 14 down-regulated) (Fig. 7, Additional file 8: Table S8).

This response is highlighted by the up-regulation of a number of chemokines (CXCL6, 11.3-fold; CXCL, 3.7fold; CXCL11, 14.4-fold) that recruit elements of the immune system including macrophages, $t$-cells and neutrophils $[45,46]$. The notable exception is the downregulation of CXCL-14 (-2.8-fold), which is thought to inhibit the signaling action of CXCL-12 and immune cell migration [47]. In addition to members of the CXCtype chemokine family, the strong up-regulation of 


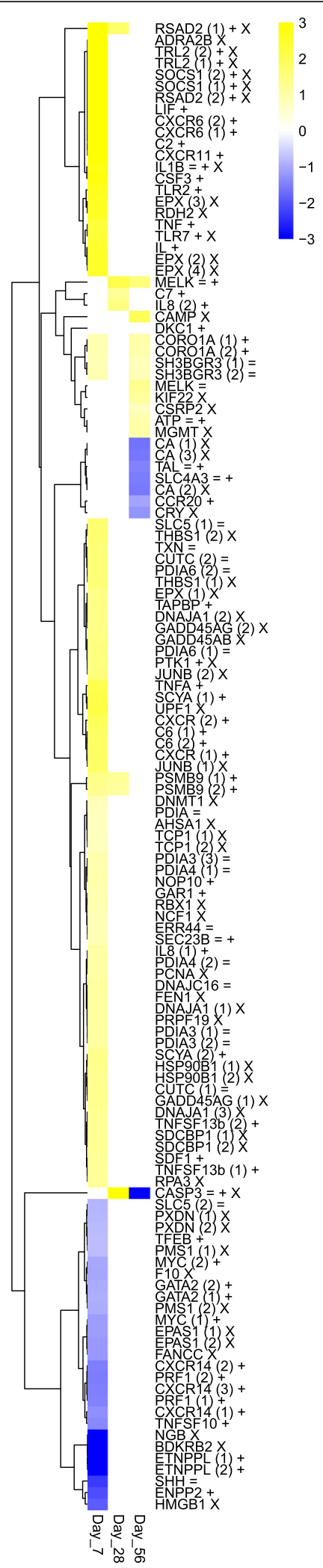

Fig. 7 Heatmap-cellular stress response: this heatmap demonstrates the change in expression patterns of genes involved in cellular stress response and homeostasis that displayed differential expression in at least one acclimation time point $(7 d, 28 d$, or $56 d)$ when fish acclimated to the multi-stressor treatment $(n=4)$ were compared to fish in the control treatments $(n=4)$. Genes associated with the gene ontology term response to stress (GO:0006950) are represented by $(\mathrm{x})$, immune system process (GO:0002376) are represented by (+), and homeostatic process (GO:0042592) are represented by (=) that displayed differential expression (FDR $\leq 0.05$ ) in at least one time point are included. Fold change is $\log _{2}$ adjusted with genes showing significant up-regulation depicted in yellow and genes showing a significant down-regulation in blue. Genes whose level of expression did not differ significantly between control and multi-stressor treatments are shown in white. The number of gene variants identified in the annotated transcriptome that were associated with the same gene name are indicated in parentheses next the gene symbol

SOCS1, which is thought to serve a vital role in both innate and adaptive immune responses [48, 49], further supports the occurrence of a robust immune response to the multi-stressor treatment at the $7 \mathrm{~d}$ time-point.

\section{Homeostatic processes}

In teleost fish, the maintenance of ionic and acid-base homeostasis are invariably linked; however, unlike terrestrial organisms, which can alter plasma $\mathrm{pH}$ by increasing or decreasing ventilation rates, acid-base balance in fish requires direct exchange of ions with the external environment via specialized chloride cells within the gill epithelium [50]. As such, when experiencing hypercapnic conditions, fish cannot "off-gas" $\mathrm{CO}_{2}$ by increasing ventilation rates. The compensation for imbalance requires the direct transfer of an acid $\left(\mathrm{H}^{+}\right)$and base $\left(\mathrm{HCO}_{3}^{-}\right)$for $\mathrm{Na}^{+}$and $\mathrm{Cl}^{-}$respectively, across the gills, kidneys and/or intestine [51-54].

We found fish acclimated to the $7 \mathrm{~d}$ multi-stressor treatment demonstrated a robust gene response associated with maintaining acid-base homeostasis. In all, 18 genes in this GO category were significantly upregulated while only 2 were significantly down-regulated (Fig. 7, Additional file 9: Table S9).

Sodium-hydrogen exchangers are known to be a primary mechanism for acid-base regulation in the gill tissue of fish, with fish excreting protons in order to achieve acid-base homeostasis when subjected to increased $\mathrm{CO}_{2}$ concentrations [55]. The acidification resulting from the increased $p \mathrm{CO}_{2}$ is likely responsible for the observed up-regulation of SLC9A5 (3.2-fold), which is a member of the sodium-hydrogen exchanger family [56]. We do observe a concurrent downregulation of SLC9A6 (-1.8-fold), however this variant is thought to localize to the mitochondria [57] and not the cellular plasma membrane where SLC9A5 is found, and thus is likely not involved in maintaining cellular $\mathrm{pH}$ counter to the external environment. 
In addition to perturbations to acid-base balance, increases in temperature and decreases in $\mathrm{pH}$ can also impact redox potential within the cell, leading to increased oxidative stress [58]. A common biomarker of oxidative stress, protein carbonyl concentrations, have previously been shown to significantly elevate in $T$. bernacchii exposed to the same multi-stressor conditions [30]. Similarly, in the expression profiles of fish in the $7 \mathrm{~d}$ multistressor treatment, we find the up-regulation of redox related proteins including TXN (thioredoxin, 2.9-fold) and SH3BGRL3 (SH3 Domain Binding Glutamate-Rich Protein Like 3, 1.9-fold).

Interestingly, a number of genes encoding for disulfide-isomerases are also up-regulated (8 gene variants up-regulated at least 1.5 fold) in the $7 \mathrm{~d}$ multistressor treatment. Protein disulfide-isomerases ("PDIs") are known to assist in proper protein folding or corrective protein folding in the ER and play key roles in the unfolded protein response (UPR) in the lumen of the ER $[59,60]$. PDIs are essential in disulfide bond formation and are likely involved in the biogenesis of a large number of membrane bound proteins necessary during cellular remodeling [61]. Their up-regulation is a further indication of the large-scale changes involving membrane bound processes such as ion regulation, acid-base balance and potentially even the redox potential of mitochondria.

\section{Pathway specific responses (28d and 56d multi-stressor treatments) \\ Carbohydrate and lipid metabolism}

mRNA expression levels for genes associated with the GO category for carbohydrate metabolism in fish acclimated for longer time-frames displayed distinct changes from the patterns observed in the $7 \mathrm{~d}$ acclimated fish (Fig. 5, Additional file 2: Table S2). Analysis of significant changes in gene expression for fish in the $28 \mathrm{~d}$ treatment groups displayed little difference between control and multi-stressor treatments while fish acclimated to the 56d multi-stressor treatment indicated a particular up-regulation of pathways involved in the catabolism of simple sugars (triosephosphate isomerase, 2.1-fold; phosphoglycerate, 2.4-fold; fructose-bisphosphate aldolase, 108.4-fold; phosphomannomutase, 1.8-fold) and the glycolytic enzyme 2-phospho-d-glycerate hydro-lyase (aka enolase, 98.6-fold). Similarly, the number of DE genes involved in lipid metabolism fell to 4 upregulated/ 0 down-regulated in the 28d fish and 6 upregulated/ 2 down-regulated in fish acclimated for $56 \mathrm{~d}$ (Fig. 5, Additional file 3: Table S3).

Following the initial cellular stress response at the $7 d$ time-point, expression of genes associated with metabolic pathways largely appeared to return to basal levels as there were very few differentially expressed genes in fish acclimated to the $28 \mathrm{~d}$ and $56 \mathrm{~d}$ multi-stressor treatments. However, the few genes that remain differentially expressed in the longer time periods of $28 \mathrm{~d}$ and $56 \mathrm{~d}$ may be evidence of the more persistent metabolic changes that are required for acclimation to elevated seawater temperatures and $p \mathrm{CO}_{2}$ levels. For example, the $56 \mathrm{~d}$ multi-stressor treatment experiences a strong up-regulation of multiple diacylglycerol kinase isoforms (2.4-fold, 3.1-fold), a potential indicator of a persistently elevated challenge in maintaining the integrity of cellular membranes. Furthermore, the heavier reliance on carbohydrate utilization and anaerobic glycolysis for ATP generation may also persist beyond the initial cellular stress response observed in the first 7 days. Indeed, this apparent change in energy usage has been shown to persist for at least 14 days in $T$. bernacchii when thermally stressed [38]. Furthermore, we have recently collected LDH activity data in these same fish that when combined with the changes in carbohydrate catabolism pathways noted above, suggests these fish may not be capable of fully compensating for the increased energetic demands of acclimating to this multi-stressor treatment [34].

\section{Cellular death and proliferation}

As seen with lipid and carbohydrate metabolism, the changes in genes associated with cellular death are also heavily diminished in the $28 \mathrm{~d}$ and $56 \mathrm{~d}$ acclimated fish (Fig. 6, Additional file 4: Table S4). At 28d few genes are differentially regulated with the notable exceptions of the down-regulation of caspase- 8 related genes $(-2.0$ to -2.7 -fold) which continued through the $56 \mathrm{~d}$ treatment ( -2.2 to -2.5 -fold decrease). The down regulation of caspase- 8 seen in the gill tissue is likely a strong indicator of a reversal of the apoptotic signal induced in the $7 \mathrm{~d}$ treatment and likely signals a return to normal cell proliferation by 28 days. Caspases are grouped into two functional families, effectors and initiators, of which caspase- 8 belongs to the latter [62]. Caspase- 8 is known to play a central role in both the cell surface-initiated apoptosis pathway as well as the mitochondrial-initiated apoptosis pathway and its activation is likely a key regulatory step in the initiation of the apoptosis signaling pathway $[63,64]$. Fish in both the $28 \mathrm{~d}$ and 56d multistressor groups appeared to exhibit dramatic changes in expression of caspase-3 precursor (+621.7-fold and -3396.9-fold, respectively). However, a closer analysis at the individual level of the expression of this particular gene indicates that these dramatic changes in expression are driven by a single individual within each treatment group, and thus are likely an aberration. The strong negative regulation of the cell proliferation also appears to be limited to the initial cellular stress response as no genes in this GO category were differentially regulated 
in the fish acclimated for $28 \mathrm{~d}$ and only one was observed in fish acclimated for 56d in the multi-stressor treatment (Fig. 7, Additional file 5: Table S5).

Our data indicate the positive regulation of cell-cycle arrest and apoptosis observed under acute thermal challenges continue for at least 7 days into a cellular stress event. Taken together with the remodeling of metabolic pathways discussed above and the physiological and biochemical analyses previously performed on these same specimens [30, 33, 34], it appears T. bernacchii requires somewhere between 14 and 28 days to transition from a bio-energetically costly cellular stress response to a state of relative cellular homeostasis. However, even after 56 days of continual exposure to a multi-stressor scenario, $T$. bernacchii may not be capable of fully compensating for elevated temperatures and $p \mathrm{CO}_{2}$ levels.

\section{Response to stress}

As seen in the $7 \mathrm{~d}$ acclimated fish, we found little evidence of the T. bernacchii is capable of mounting an effective heat shock response in gill tissue after chronic exposure to a stressful environment. In the fish acclimated to the $28 \mathrm{~d}$ multi-stressor treatment we observed zero DE genes in this GO category and only 3 in fish acclimated to the multi-stressor treatment for 56d (Fig. 7, Additional file 6: Table S6). We did however note a conspicuous -4.5 -fold decrease in transcript levels for the constitutively expressed chaperone, HSC71 after 56d of acclimation to ${ }^{+} 4{ }^{\circ} \mathrm{C}$ and $1000 \mu \mathrm{ATM} p \mathrm{CO}_{2}$ (Fig. 7, Additional file 6: Table S6). These data are consistent with results obtained from a previous description of the de novo transcriptome assembly of $T$. bernacchii in which we noted a down-regulation of constitutively expressed chaperones in a number of tissues after a $28 \mathrm{~d}$ acclimation to ${ }^{+} 4{ }^{\circ} \mathrm{C}$ alone [26]. Bilyk and Cheng confirmed a similar trend in liver tissues of $P$. borchgrevinki after a short-term acclimation (2-4 days) to elevated temperatures [25].

These changes in constitutive chaperones also coincide with reduced levels of oxidative damage in the gill tissue of both T. bernacchii and P. borchgrevinki [30]. Taken together, these unexpected shifts in chaperoning capacity and protein damage suggest acclimation to elevated temperature may have interesting and perhaps unexpected consequences for protein dynamics in these fish.

\section{Immune system processes}

Unlike many of the other GO sub-categories assessed in this study, a more sustained differential gene expression response was observed within immune system processes (7 up-regulated at 28d; 5 up-regulated, 4-down regulated at 56d) (Fig. 7, Additional file 8: Table S8). While it may be possible that the immune system is responding to a latent infection that is able to manifest due to the effects of the multi-stressor treatment on $T$. bernacchii, the more likely scenario is that the observed response is to wide spread cellular damage and remodeling directly resulting from the multi-stress treatment.

This argument is supported by indicators of cellular damage, such as PSMB9, which is also up-regulated at the $7 \mathrm{~d}$ (2.5-fold) and $28 \mathrm{~d}$ (2.3-fold) time-points. PSMB9, also known as $20 \mathrm{~S}$ proteasome subunit $\beta-1 \mathrm{i}$, is an essential subunit of the proteasome and may be associated with a more specialized form of the proteasome known as the immune proteasome [65]. PSMB9 is required for the production of MHC class-1 restricted T-cell epitopes, and thus plays an important role in antigen processing. Furthermore, a previous study measuring stress at the cellular level found that several notothenioids exhibit considerable increases in oxidative damage after 7 days of heat and $p \mathrm{CO}_{2}$ stress, which diminishes at 28 and 56 days [30]; indicating a sufficient level of protein damage likely exists to result in the activation of the immune proteasome seen here.

\section{Homeostatic processes}

The concurrent up-regulation of $\mathrm{pH}$ regulators, redox regulators and disulfide isomerases observed at the 7 day time-point in the multi-stressor condition is indicative of the challenges $T$. bernacchii faces in maintaining homeostasis under these conditions. After the short-term acclimation response, $T$. bernacchii no longer experiences dramatic changes in expression of proteins related to the maintenance of cellular homeostasis. As seen with previous categories, this response tapered off rapidly at $28 \mathrm{~d}$ with only 2 up-regulated genes, suggesting these fish are capable of at least partially compensating for the changes in extracellular $\mathrm{pH}$ and osmolarity likely caused by the multi-stressor condition (Fig. 7). A notable exception is the continued up-regulation of SH3BGRL3 throughout the 56d time point (Fig. 7), which may indicate the continued need for assisted folding of membrane associated proteins under the multi-stressor condition and may be representative of a protracted cost to cellular maintenance. Despite the return of most gene expression patterns to a basal state observed in control fish, the continued upregulation of some key cellular functions provides further support that $T$. bernacchii may not be fully capable of compensating for these experimental conditions within the $56 \mathrm{~d}$ acclimation period studied here.

\section{Conclusion}

Our efforts have uncovered several key findings concerning the molecular response of $T$. bernacchii under the multi-stressor condition brought on by increased sea surface temperature and ocean acidification. First, although previous studies using acute thermal stress suggest a portion of the conserved cellular stress response 
is retained in these fish, we have now shown that $T$. bernacchii is capable of mounting a robust and coordinated response to stress and that this response persists for at least 7 days and perhaps as long as 2 weeks. Among the conserved responses observed were DNA repair pathways accompanied by cell-cycle arrest and apoptosis; maintenance of acid-base balance and redox-potential; and cytokine signaling and cellular inflammation. As found in previous studies under heat stress alone, $T$. bernacchii does not possess an inducible heat shock response when exposed to synergistic heat and $p \mathrm{CO}_{2}$ stress. However, the up-regulation of chaperones and PDIs localized in the ER may indicate the unfoldedprotein response of the ER remains intact in these fish.

Second, the multi-stressor treatment results in significant cellular damage that continues for at least 7 days after the initial exposure. In addition to the increase in oxidatively damaged proteins previously noted in these fish, there also appears to be a significant amount of DNA damage accrued as indicated by the up-regulation of pathways associated with cellular death, DNA damage responses, and immune system responses. Furthermore, the multi-stressor treatment induces a strong initial response that likely comes at a significant energetic cost to the organism. In addition to the activation of stress responsive pathways, we also observed changes in gene expression patterns that suggest a shift in substrate preference for glycolysis and the possible reliance on anaerobic pathways to supplement ATP generation.

Our findings demonstrate that the multi-stressor condition induces a strong short-term response that returns to near basal levels of expression within most of the studied pathways, indicating some degree of compensation to the environmental changes has set in. This would seem to indicate that $T$. bernacchii possesses the physiological plasticity to cope with an environment similar to the multi-stressor condition and thus also with the changing climate of the Southern Ocean.

Although our study demonstrates that after 56 days of exposure to the multi-stressor condition, expression levels across the transcriptome largely returned to near basal levels, it is noteworthy that not all gene expression patterns returned to control levels and this may be an indication that $T$. bernacchii has not fully compensated within the 56d acclimation period. We identifed subtle changes in expression that persist through the 56d timepoint (such as shifts towards carbohydrate metabolism and continued maintenance of membrane protein homeostasis) that may significantly affect $T$. bernacchii's fitness over a longer period of time. Furthermore, in a related study, we tracked growth parameters and calculated Fulton's index for these fish over the course of the experiment and found temperature had a negative impact on fish condition. In particular, $T$. bernacchii displayed significant declines in condition when acclimated to $4{ }^{\circ} \mathrm{C}$ for $28 \mathrm{~d}$ or longer [34]. As such, we must approach conclusions about their capacity to adapt to future ocean conditions with caution, as this physiological plasticity does not appear to rise to the level of warm hardiness previously observed in other notothenioid species [36]. Figure 8 provides a summary schematic of the molecular responses observed over the 56 day acclimation period and how they may scale up to impact the physiology of these fish in a high $\mathrm{CO}_{2}$ ocean.

Lastly, as this study focused upon the gills of adult fish only, and did not investigate juveniles, other tissues, or the overall reproductive cycle; it is difficult to surmise the overall effect that these synergistic stressors may have on the fitness of $T$. bernacchii at the population level and over the course of many generations. Further inquiry is necessary to address these concerns, and to further elucidate $T$. bernacchii's potential to adapt to changing environmental conditions.

\section{Methods \\ Collection of fish}

Specimens of T. bernacchii were collected in McMurdo Sound, Antarctica from September through December, 2012. Fish were caught using hook and line through 10-in. holes drilled through the sea ice and transported back to McMurdo Station in aerated coolers where they were housed in flow-through aquaria maintained at ambient seawater temperature $\left(-1.5{ }^{\circ} \mathrm{C}\right)$. Fish were then tankacclimated under ambient conditions for one week prior to being placed in experimental tanks. All procedures were conducted in accordance with the Animal Welfare Act and were approved by the University of South Carolina Institutional Animal Care and Use Committee (ACUP protocol \# 100377).

\section{Experimental design}

We used four, $1240 \mathrm{~L}$ experimental tanks to assess the combined effects of elevated temperature and $p \mathrm{CO}_{2}$ on T. bernacchii. Our two experimental treatments consisted of a control tank which was held near ambient conditions $\left(-1{ }^{\circ} \mathrm{C}\right.$ and $\left.430 \mu \mathrm{atm}\right)$ and a high temperature + high $p \mathrm{CO}_{2}$ treatment $\left(+4{ }^{\circ} \mathrm{C} / 1000 \mu \mathrm{atm}\right)$. Fish were placed in experimental tanks and acclimated for a total of 56 days. Five fish per treatment were removed at $7 \mathrm{~d}, 28 \mathrm{~d}$, and $56 \mathrm{~d}$ time-points, after which fish were sacrificed and gill tissues were collected and immediately flash-frozen in liquid nitrogen. Although we recognize a fully replicated experimental design is ideal to exclude tank effects as a possible confounding factor, the constraints of working in Antarctica prevented us from using this approach. However, our previous analyses show no tank effect when treatments were alternated between tanks across multiple seasons $[30,33]$. 


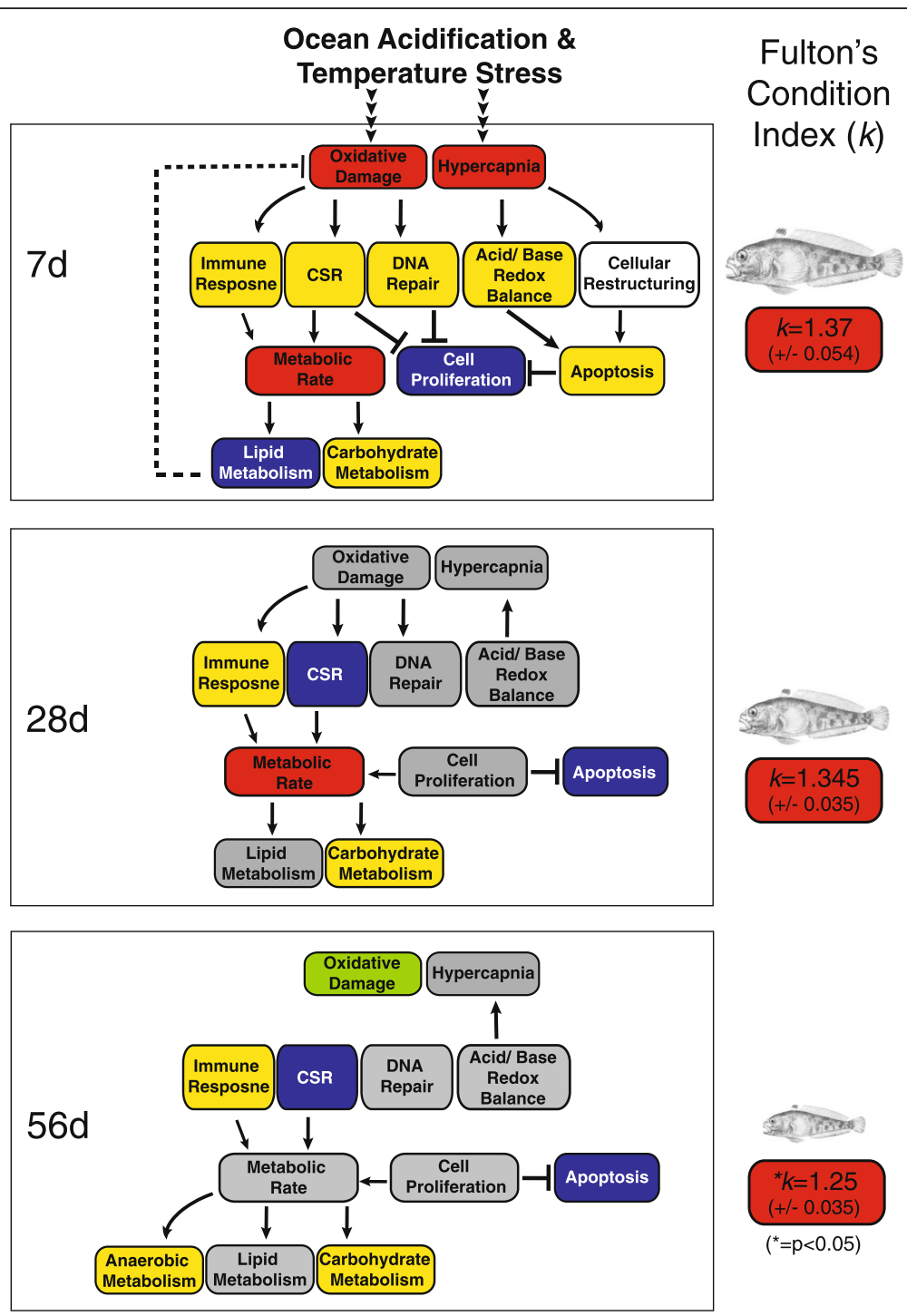

Fig. 8 Schematic of molecular response integrated with physiological changes at the organismal level: Gene expression patterns identified in this study suggest a number of cellular pathways that were up-regulated (yellow boxes) or down-regulated (blue boxes) in fish acclimated to combined elevated temperature and $\mathrm{PCO}_{2}$ stressors over the course of 56 days. These changes likely underlie the physiological changes previously observed in $T$. bernacchii under these multi-stressor conditions (red boxes [30, 33, 34]). Although the expression of most genes returned to basal levels, a number of key genes remained up-regulated at the 56 day time-point suggesting T. bernacchii has not fully compensated. Ultimately, these stressors may impact the fish condition overall (as suggested by Fulton's condition index [34]) and may have long-term population level impacts such as reduced growth and reproduction

\section{Manipulation of seawater conditions}

Temperature and $p \mathrm{CO}_{2}$ levels were manipulated within the experimental treatment tanks using a $p \mathrm{CO}_{2}$ generation system first described by Fangue et al. [66] and adapted for use with large-scale applications and combined with thermostated titanium heaters (Process Technology, Brookfield CT, USA; Enzor et al. [33]). Atmospheric air was pumped through drying columns (filled with drierite) to remove moisture, and air was scrubbed of $\mathrm{CO}_{2}$ using columns filled with Sodasorb. Pure $\mathrm{CO}_{2}$ and $\mathrm{CO}_{2}$-free air were then blended using digital mass flow controllers and bubbled into header tanks that were continuously replenished with ambient seawater using venturri injectors, which in turn fed into experimental treatment tanks.

Temperature, $\mathrm{pH}$ (total scale), salinity, total alkalinity $\left(T_{A}\right)$ and oxygen saturation were measured daily from both incoming seawater as well as experimental treatment tanks. For $p \mathrm{CO}_{2}$ analysis, we followed the SOP as described in the Best Practices Guide [67] for the spectrophotometric determination of $\mathrm{pH}$ using $\mathrm{m}$-cresol purple and measurement of total alkalinity via acid titration using a computer-controlled T50 Titrator (Mettler Toledo, Columbus, $\mathrm{OH}, \mathrm{USA})$. Temperature was measured with a 
calibrated digital thermocouple (Omega Engineering Inc., Stamford, CT, USA) and salinity was measured using a YSI 3100 Conductivity meter (Yellow Springs, OH, USA). $\mathrm{CO}_{2}$ calc [68], using the constants of Mehrbach et al. [69] as refit by Dickson and Millero [70], was used to calculate all other carbonate parameters. Oxygen saturation was recorded using a galvanic oxygen probe (Loligo Systems, Denmark). Mean values ( \pm s.d.) of temperature $\left({ }^{\circ} \mathrm{C}\right)$ and $p \mathrm{CO}_{2}$ ( $\left.\mu \mathrm{atm}\right)$ over the course of the experiment were first reported by Enzor and Place [30]. Additionally, treatment tanks were sampled daily for the presence of ammonia, nitrite and nitrates, with no significant increase in waste products noted over the course of the experiment (data not shown).

\section{Tissue collection and RNA extraction}

To obtain individual gill-specific expression profiles, we separately indexed and sequenced RNA samples from gill tissue that had been collected from fish acclimated to the two experimental treatments described above $(n=5$ fish per treatment) for 7, 28 and 56 days. Immediately after euthanizing the fish, tissues were excised in a $-2{ }^{\circ} \mathrm{C}$ environmental chamber, flash frozen in liquid nitrogen, and shipped back to our home institution on dry ice where they were stored at $-80{ }^{\circ} \mathrm{C}$ until used. Total RNA from approximately $100 \mathrm{mg}$ of frozen tissue was extracted using TRIzol (Invitrogen) following the manufacturer's recommendations. The RNA was further cleaned by re-suspending in $0.1 \mathrm{ml}$ of RNase/ DNasefree water and adding $0.3 \mathrm{ml}$ of $6 \mathrm{M}$ guanidine $\mathrm{HCl}$ and $0.2 \mathrm{ml}$ of $100 \%$ ethylalcohol (EtOH). The entire volume was loaded onto a spin column (Ambion) and centrifuged for $1 \mathrm{~min}$ at $12,000 \times \mathrm{g}$ at $4{ }^{\circ} \mathrm{C}$. Flow-through was discarded, and filters were washed twice with $0.2 \mathrm{ml} 80 \% \mathrm{EtOH}$. RNA was eluted off of the filters twice with $0.1 \mathrm{ml}$ of DEPC-treated water. RNA was precipitated by the addition of $0.1 \mathrm{vol}$ of $3 \mathrm{M}$ sodium acetate ( $\mathrm{pH} 5.0)$ and $2.5 \mathrm{vol}$ of $100 \% \mathrm{EtOH}$, mixed by inversion of tubes and placed at $-80{ }^{\circ} \mathrm{C}$ for $1 \mathrm{~h}$. After this period, tubes were centrifuged at $12,000 \times \mathrm{g}$ for $20 \mathrm{~min}$ at $4{ }^{\circ} \mathrm{C}$. Pellets were washed twice with $80 \%$ $\mathrm{EtOH}$ and re-suspended in $30 \mu \mathrm{l}$ of RNase/ DNase-free water. Lastly, RNA was DNase treated at $30{ }^{\circ} \mathrm{C}$ for 10 min. Total RNA from $n=5$ fish within an acclimation treatment was submitted to the Vaccine and Gene Therapy Institute (VGTI) Florida for quality assessment and determination of specific concentration using an Agilent 2100 BioAnalyzer. From the original samples, the 4 highest quality replicates from each treatment and time point were selected for cluster generation using the Illumina ${ }^{\circ}$ TruSeq RNA Sample Prep v2 Hs Protocol and sequencing via an Illumina ${ }^{\circ}$ HiSeq 2500 Rapid Run initialized for single-end 100 bp reads.

\section{Sequencing read quality control and mapping}

Raw reads from each of the 24 samples were processed using Trimmomatic [27]. Illumina ${ }^{\circ}$ TruSeq RNA Sample Prep v2 HS adapters were removed as well as any bases on the end of the reads with a PHRED33 score of $<20$ or any portion of the read that did not average at least a PHRED33 score $>20$ across a minimum span of 4 bp (Trimmomatic parameter input: ILLUMINACLIP:Trimmomatic-0.32/adapters/TruSeq2-SEMultiplex.fa:2:30:10 LEADING:20 TRAILING:20 SLIDINGWINDOW:4:20 MINLEN:75). Following trimming, only sequencing reads $\geq 75$ bp in length were retained. The remaining sequencing reads were individually mapped to an existing transcriptome using Bowtie2-2.2.3 with standard parameters [28]. The transcriptome employed as a reference was an enhancement of the previously published T. bernacchii transcriptome [26] using 150 bp pairedend Illumina reads as additional input into the Trinity Assembly program [71] under default parameters. Assembly was conducted on the XSEDE Blacklight Supercomputing Resource utilizing the native Trinity module [72]. The additional Illumina reads were obtained from pooled RNA isolated from the gill, liver and brain tissue of $T$. bernacchii. After assembly the transcriptome was pruned using RSEM [73] with any transcripts with expression values less than 0.001 FPKM removed, and then further compacted using CD-HIT-EST [74] at a percent identity of $100 \%$. Annotation was facilitated by the XSEDE Stampede Supercomputer [75] utilizing the native ncbi-Blast module in a massively parallel structure [76] and then completed on local resources with the BLAST2GO command line utility [77].

\section{Differential gene expression analysis}

Using raw mapping counts from Bowtie2 [28], RNA-Seq by Expectation-Maximization ("RSEM" version 1.2.18) [75] analyses were conducted to generate estimated read-count (count) and fragments per kilobase million (FPKM) counts for each sample at the transcript and gene level. The Trinity pipeline (Trinity version trinityrnaseq_r20140717) [78] was used to aggregate these counts into master matrices for import into the $R$ statistical package [79]. Before import, the samples were grouped by time-point and treatment similarity to conduct pairwise analyses of the effect of the multistressor treatment over time as compared to the control. Empirical analysis of digital gene expression data in $R$ ("edgeR" version 3.4.2) was implemented to conduct differential gene expression analyses [29]; dispersion values were calculated using the replicate groups; and exact tests utilizing a negative binomial distribution with a cutoff false discovery rate of 0.05 were used to identify differentially expressed transcripts and genes. 
A sample-level differential expression heat map was generated from the differential gene expression analyses resulting from edgeR using the Trinity pipeline. Within the BLAST2GO graphical interface package [80] Fisher's Exact tests (FDR $\leq 0.05)$ were conducted for differentially expressed transcripts of the multi-stressor treatment to identify over-represented gene ontology terms within the up- and down- regulated transcripts within each treatment group in general. Using custom Python scripts annotation and expression data files were combined, and GO categories and genes of interest were extracted for further analysis.

\section{Availability of supporting data}

The raw data were deposited at the NCBI Sequence Read Archive under the Bioproject accession number PRJNA289753.

\section{Additional files}

Additional file 1: Table S1. GO over representation analysis: This analysis table contains the raw data for the identification of $\mathrm{GO}$ terms over-represented in the $7 \mathrm{~d}$ multi-stressor up-regulated, $7 \mathrm{~d}$ multi-stressor down-regulated, 28d multi-stressor up-regulated, 28 multi-stressor down-regulated, 56 multi-stressor up-regulated, and 56 multi-stressor down-regulated subgroups. The table provides the GO-ID, GO Term, and number of terms found if over-represented in the subgroup (a blank entry indicates a lack of significance for that $\mathrm{GO}$ term). The total number of each $\mathrm{GO}$ terms found in the reference transcriptome is also included. (XLSX $21 \mathrm{~kb}$ )

Additional file 2: Table S2. Carbohydrate metabolic process: This table provides all differentially expressed genes exceeding the confidence threshold (FDR $\leq 0.05$ ) that are associated with the GO Term carbohydrate metabolic process (GO:0005975) at 7d, $28 \mathrm{~d}$ and $56 \mathrm{~d}(n=4)$. The table includes the gene sequence name from the reference transcriptome, the BLAST result description, the sequence length, the number of BLAST hits to that gene sequence, the minimum e-value of the BLAST hits, the mean similarity, the \# of GO terms associated with that sequence, the list of those GO terms, the Enzyme Codes associated with that sequence, Interproscan results associated with that sequence, the $\log _{2}$ adjusted fold change in expression, the $\log _{2}$ counts per million (CPM) of the reads mapped to that sequence, and the $p$-value and FDR reflecting the confidence in the displayed differential expression of that gene. (XLSX $23 \mathrm{~kb}$ )

Additional file 3: Table S3. Lipid metabolic process: This table provides all differentially expressed genes exceeding the confidence threshold (FDR $\leq 0.05$ ) that are associated with the GO Term lipid metabolic process (GO:0006629) at 7d, 28d and $56 \mathrm{~d}(n=4)$. The table includes the gene sequence name from the reference transcriptome, the BLAST result description, the sequence length, the number of BLAST hits to that gene sequence, the minimum e-value of the BLAST hits, the mean similarity, the \# of GO terms associated with that sequence, the list of those GO terms, the Enzyme Codes associated with that sequence, Interproscan results associated with that sequence, the $\log _{2}$ adjusted fold change in expression, the $\log _{2}$ counts per million (CPM) of the reads mapped to that sequence, and the $p$-value and FDR reflecting the confidence in the displayed differential expression of that gene. (XLSX $20 \mathrm{~kb}$ )

Additional file 4: Table S4. Cell death: This table provides all differentially expressed genes exceeding the confidence threshold $(F D R \leq 0.05)$ that are associated with the GO Term cell death (GO:0008219) at $7 \mathrm{~d}, 28 \mathrm{~d}$ and $56 \mathrm{~d}(n=4)$. The table includes the gene sequence name from the reference transcriptome, the BLAST result description, the sequence length, the number of BLAST hits to that gene sequence, the minimum e-value of the BLAST hits, the mean similarity, the \# of GO terms associated with that sequence, the list of those GO terms, the Enzyme Codes associated with that sequence, InterproScan results associated with that sequence, the $\log _{2}$ adjusted fold change in expression, the $\log _{2}$ counts per million (CPM) of the reads mapped to that sequence, and the $p$-value and FDR reflecting the confidence in the displayed differential expression of that gene. (XLSX $17 \mathrm{~kb})$

Additional file 5: Table S5. Cell proliferation: This table provides all differentially expressed genes exceeding the confidence threshold (FDR $\leq 0.05)$ that are associated with the GO Term cell proliferation (GO:0008283) at 7d, 28d and 56d $(n=4)$. The table includes the gene sequence name from the reference transcriptome, the BLAST result description, the sequence length, the number of BLAST hits to that gene sequence, the minimum e-value of the BLAST hits, the mean similarity, the \# of GO terms associated with that sequence, the list of those GO terms, the Enzyme Codes associated with that sequence, Interproscan results associated with that sequence, the $\log _{2}$ adjusted fold change in expression, the $\log _{2}$ counts per million (CPM) of the reads mapped to that sequence, and the $p$-value and FDR reflecting the confidence in the displayed differential expression of that gene. (XLSX $14 \mathrm{~kb}$ )

Additional file 6: Table S6. Heat shock related proteins: This table provides all genes associated with heat shock proteins found within the reference transcriptome regardless of expression/confidence level at $7 d$, $28 \mathrm{~d}$ and $56 \mathrm{~d}(n=4)$. Each time-point sub-table is sorted by FDR (smallest first). The table includes the gene sequence name from the reference transcriptome, the BLAST result description, the sequence length, the number of BLAST hits to that gene sequence, the minimum e-value of the BLAST hits, the mean similarity, the \# of GO terms associated with that sequence, the list of those GO terms, the Enzyme Codes associated with that sequence, InterproScan results associated with that sequence, the $\log _{2}$ adjusted fold change in expression, the $\log _{2}$ counts per million (CPM) of the reads mapped to that sequence, and the $p$-value and FDR reflecting the confidence in the displayed differential expression of that gene. (XLSX 37 kb)

Additional file 7: Table S7. Response to stress: This table provides all differentially expressed genes exceeding the confidence threshold $(F D R \leq 0.05)$ that are associated with the GO Term response to stress (GO:0006950) at 7d, 28d and 56d ( $n=4)$. The table includes the gene sequence name from the reference transcriptome, the BLAST result description, the sequence length, the number of BLAST hits to that gene sequence, the minimum e-value of the BLAST hits, the mean similarity, the \# of GO terms associated with that sequence, the list of those GO terms, the Enzyme Codes associated with that sequence, InterproScan results associated with that sequence, the $\log _{2}$ adjusted fold change in expression, the $\log _{2}$ counts per million (CPM) of the reads mapped to that sequence, and the $p$-value and FDR reflecting the confidence in the displayed differential expression of that gene. (XLSX 26 kb)

Additional file 8: Table S8. Immune system process: This table provides all differentially expressed genes exceeding the confidence threshold (FDR $\leq 0.05$ ) that are associated with the GO Term immune system process (GO:0002376) at $7 \mathrm{~d}, 28 \mathrm{~d}$ and $56 \mathrm{~d}(n=4)$. The table includes the gene sequence name from the reference transcriptome, the BLAST result description, the sequence length, the number of BLAST hits to that gene sequence, the minimum e-value of the BLAST hits, the mean similarity, the \# of GO terms associated with that sequence, the list of those GO terms, the Enzyme Codes associated with that sequence, InterproScan results associated with that sequence, the $\log _{2}$ adjusted fold change in expression, the $\log _{2}$ counts per million (CPM) of the reads mapped to that sequence, and the $p$-value and FDR reflecting the confidence in the displayed differential expression of that gene. (XLSX $23 \mathrm{~kb}$ )

Additional file 9: Table S9. Homeostatic process: This table provides all differentially expressed genes exceeding the confidence threshold $(F D R \leq 0.05)$ that are associated with the GO Term homeostatic process (GO:0042592) at 7d, 28d and 56d ( $n=4)$. The table includes the gene sequence name from the reference transcriptome, the BLAST result description, the sequence length, the number of BLAST hits to that gene sequence, the minimum e-value of the BLAST hits, the mean similarity, the \# of GO terms associated with that sequence, the list of those GO terms, the Enzyme Codes associated with that sequence, Interproscan results associated with that sequence, the $\log _{2}$ adjusted fold change in expression, the $\log _{2}$ counts per million (CPM) of the reads mapped to that sequence, and the $p$-value and FDR reflecting the confidence in the displayed differential expression of that gene. (XLSX $15 \mathrm{~kb}$ ) 


\section{Abbreviations}

bp: Base pair; CHR: Cellular homeostasis response; CS: Citrate synthase; CSR: Cellular stress response; Cyt-c: cytochtome-c oxidase; ER: Endoplasmic reticulum; FDR: False discovery rate; FPKM: Fragments per kilobase million; GO: Gene Ontology; HOAD: Hydroxyacyl-CoA dehydrogenase; IPCC: Intergovernmental Panel on Climate Change; LDH: Lactate dehydrogenase; $\mathrm{CCO}_{2}$ : Partial pressure carbon dioxide; RNA-Seq: RNA sequencing; RSEM: RNA-Seq by Expectation-Maximization; SST: Sea surface temperature; UPR: Unfolded protein response.

\section{Competing interests}

The authors declare no competing interests.

\section{Authors' contributions}

SP designed the research and performed the experiments. TH prepared samples for sequencing and analyzed the data. TH and SP wrote the article. Both authors read and approved the final manuscript.

\section{Acknowledgements}

There are many people who assisted us during the course of this project. In particular, we thank Dr. Jeff Dudycha, Dr. Mackenzie Zippay, Evan Hunter and Allison "Sandwich" Barden for their help collecting specimens and with seawater chemistry measurements. We would like to further thank Dr. Jeff Dudycha for his advisement throughout the analysis process and helpful comments during the writing of this manuscript. We would also like to thank the United States Antarctic Program, Lockheed Martin Antarctic Support Contract, and the countless number of USAP support personnel for their tireless logistical and field support while at McMurdo Station. This work used the Extreme Science and Engineering Discovery Environment (XSEDE), which is supported by National Science Foundation grant numbers $\mathrm{ACl}-1053575$ and $\mathrm{OCl}-1053575$. Specifically, we would like to thank the teams managing the Blacklight system at the Pittsburgh Supercomputing Center and the Stampede system at the Texas Advanced Computing Center for assisting our efforts in high performance computing. This research was supported by National Science Foundation (NSF) Grants PLR-1040495 and PLR-1447291 to SPP

\section{Author details}

'Department of Biology, Sonoma State University, Rohnert Park, CA 94928, USA. '2Department of Biological Sciences, University of South Carolina, Columbia, SC 29208, USA.

Received: 1 August 2015 Accepted: 9 February 2016 Published online: 20 February 2016

\section{References}

1. Peck LS, Barnes DKA, Willmott J. Responses to extreme seasonality in food supply: diet plasticity in Antarctic brachiopods. Mar Biol. 2005;147:453-63.

2. Rogers AD, Murphy EJ, Johnston NM, Clarke A. Introduction. Antarctic ecology: from genes to ecosystems. Part 2. Evolution, diversity and functional ecology. Philos Trans R Soc Lond B Biol Sci. 2007:362:2187-9.

3. Somero GN. The physiology of climate change: how potentials for acclimatization and genetic adaptation will determine 'winners' and 'losers'. J Exp Biol. 2010;213:912-20.

4. Tillmann P. Climate change effects and adaptation approaches in marine and coastal ecosystems of the North Pacific Landscape Conservation Cooperative Region. 2011. p. 279. at http://pajk.arh.noaa.gov/Articles/ articles/NPLCC_MarineClimateEffects.pdf

5. Portner $\mathrm{HO}$, Knust R. Climate change affects marine fishes through the oxygen limitation of thermal tolerance. Science. 2007;315:95-7.

6. Solomon S et al. Contribution of Working Group III to the fourth assessment report of the Intergovernmental Panel on Climate Change. Climate change 2007: the physical science basis. 2007. at http://philpapers.org/rec/METCOW.

7. Hoegh-guldberg O, Bruno JF. The impact of climate change on the world's marine ecosystems. Science. 2010;328:1523-8.

8. Gon O, Heemstra P. Fishes of the Southern Ocean. Grahamstown, South Africa: J L B Smith Institute of Ichthyology; 1990.

9. Somero GN, DeVries AL. Temperature tolerance of some Antarctic fishes. Science. 1967;156:257-8.
10. Forster ME, Franklin CE, Taylor HH, Davison W. The aerobic scope of Antarctic fish. Pagothenia borchgrevinki and it significance for metabolic cold adaptation. Polar Biol. 1987;8:155-9.

11. Davison W, Franklin CE, Carey PW. Oxygen uptake in the Antarctic teleost Pagothenia borchgrevinki. Limitations imposed by X-cell gill disease. Fish Physiol Biochem. 1990;8:69-77.

12. Pörtner HO, Lucassen M, Storch D. Metabolic biochemistry: its role in thermal tolerance and in the capacities of physiological and ecological function. Fish Physiology. 2005;22:79-154.

13. Robinson E, Davison W. The Antarctic notothenioid fish Pagothenia borchgrevinki is thermally flexible: acclimation changes oxygen consumption. Polar Biol. 2008;31:317-26.

14. Bilyk KT, Evans CW, DeVries AL. Heat hardening in Antarctic notothenioid fishes. Polar Biol. 2012;35:1447-51.

15. Bilyk KT, Cheng CHC. Model of gene expression in extreme cold-reference transcriptome for the high-Antarctic cryopelagic notothenioid fish Pagothenia borchgrevinki. BMC Genomics. 2013;14:634

16. Bilyk KT, DeVries AL. Heat tolerance and its plasticity in Antarctic fishes. Comp Biochem Physiol - A Mol Integr Physiol. 2011;158:382-90.

17. Schulte PM. Responses to environmental stressors in an estuarine fish: Interacting stressors and the impacts of local adaptation. J Therm Biol. 2007;32:152-61.

18. Rosa R, Seibel BA. Synergistic effects of climate-related variables suggest future physiological impairment in a top oceanic predator. Proc Natl Acad Sci U S A. 2008;105:20776-80

19. O'Donnell MJ, Hammond LM, Hofmann GE. Predicted impact of ocean acidification on a marine invertebrate: elevated $\mathrm{CO} 2$ alters response to thermal stress in sea urchin larvae. Mar Biol. 2009;156:439-46.

20. Hofmann GE, Todgham AE. Living in the now: physiological mechanisms to tolerate a rapidly changing environment. Annu Rev Physiol. 2010;72:127-45.

21. Gutt J et al. The Southern Ocean ecosystem under multiple climate change stresses-an integrated circumpolar assessment. Glob Chang Biol. 2014. doi: 10.1111/gcb.12794.

22. Place SP, O'Donnell MJ, Hofmann GE. Gene expression in the intertidal mussel Mytilus californianus: physiological response to environmental factors on a biogeographic scale. Mar Ecol Prog Ser. 2008;356:1-14.

23. Buckley BA, Gracey AY, Somero GN. The cellular response to heat stress in the goby Gillichthys mirabilis: a CDNA microarray and protein-level analysis. J Exp Biol. 2006:209:2660-77.

24. Jia D, Jurkowska RZ, Zhang X, Jeltsch A, Cheng X. Structure of Dnmt3a bound to Dnmt3L suggests a model for de novo DNA methylation. Nature. 2007;449:248-51.

25. Bilyk KT, Cheng CHC. RNA-seq analyses of cellular responses to elevated body temperature in the high Antarctic cryopelagic nototheniid fish Pagothenia borchgrevinki. Mar Genomics. 2014;18:163-71.

26. Huth TJ, Place SP. De novo assembly and characterization of tissue specific transcriptomes in the emerald notothen, Trematomus bernacchii. BMC Genomics. 2013;14:805.

27. Bolger AM, Lohse M, Usadel B. Trimmomatic: a flexible trimmer for Illumina sequence data. Bioinformatics. 2014. doi:10.1093/bioinformatics/btu170.

28. Langmead B, Salzberg SL. Fast gapped-read alignment with Bowtie 2. Nat Methods. 2012;9:357-9.

29. Robinson MD, McCarthy DJ, Smyth GK. edgeR: a Bioconductor package for differential expression analysis of digital gene expression data. Bioinformatics. 2010;26:139-40.

30. Enzor LA, Place SP. Is warmer better? Decreased oxidative damage in notothenioid fish after long-term acclimation to multiple stressors. J Exp Biol. 2014;217:3301-10.

31. Buckley BA, Somero GN. CDNA microarray analysis reveals the capacity of the cold-adapted Antarctic fish Trematomus bernacchii to alter gene expression in response to heat stress. Polar Biol. 2009;32:403-15.

32. Kültz D. Molecular and evolutionary basis of the cellular stress response. Annu Rev Physiol. 2005;67:225-57.

33. Enzor LA, Zippay ML, Place SP. High latitude fish in a high $\mathrm{CO} 2$ world: synergistic effects of elevated temperature and carbon dioxide on the metabolic rates of Antarctic notothenioids. Comp Biochem Physiol A Mol Integr Physiol. 2013;164:154-61.

34. Enzor LA, Place SP. The effects of elevated temperature and ocean acidification on the metabolic pathways of notothenioid fish. (Unpub Data currently in review).

35. Crockett EL, Sidell BD. Some pathways of energy metabolism are cold adapted in Antarctic fishes. Physiol Zool. 1990;63:472-88. 
36. Seebacher F, Davison W, Lowe CJ, Franklin CE. A falsification of the thermal specialization paradigm: compensation for elevated temperatures in Antarctic fishes. Biol Lett. 2005;1:151-4.

37. Windisch HS, Kathover R, Portner H-O, Frickenhaus S, Lucassen M. Thermal acclimation in Antarctic fish: transcriptomic profiling of metabolic pathways. AJP Regul Integr Comp Physiol. 2011;301:R1453-66.

38. Jayasundara N, Healy TM, Somero GN. Effects of temperature acclimation on cardiorespiratory performance of the Antarctic notothenioid Trematomus bernacchii. Polar Biol. 2013;36:1047-57.

39. Sleadd IM et al. Sub-lethal heat stress causes apoptosis in an Antarctic fish that lacks an inducible heat shock response. J Therm Biol. 2014;44:119-25.

40. Hofmann GE, Buckley BA, Airaksinen S, Keen JE, Somero GN. Heat-shock protein expression is absent in the antarctic fish Trematomus bernacchii (family Nototheniidae). J Exp Biol. 2000;203:2331-9.

41. Buckley BA, Place SP, Hofmann GE. Regulation of heat shock genes in isolated hepatocytes from an Antarctic fish, Trematomus bernacchii. J Exp Biol. 2004;207:3649-56.

42. Basu $\mathrm{N}$ et al. Heat shock protein genes and their functional significance in fish. Gene. 2002;295:173-83.

43. Liebermann DA, Hoffman B. Gadd45 in stress signaling. J Mol Signal. 2008:3:15.

44. Wendelaar Bonga SE. The stress response in fish. Physiol Rev. 1997;77:591-625.

45. Wuyts $A$ et al. Characterization of synthetic human granulocyte chemotactic protein 2: usage of Chemokine receptors CXCR1 and CXCR2 and in vivo inflammatory properties. Biochemistry. 1997;36:2716-23.

46. Cole KE et al. Interferon-inducible T cell alpha chemoattractant (I-TAC): a novel non-ELR CXC chemokine with potent activity on activated T cells through selective high affinity binding to CXCR3. J Exp Med. 1998;187:2009-21.

47. Hara T, Tanegashima K. CXCL14 antagonizes the CXCL12-CXCR4 signaling axis. Biomol Concepts. 2014:5:167-73.

48. Ben-Zvi T, Yayon A, Gertler A, Monsonego-Ornan E. Suppressors of cytokine signaling (SOCS) 1 and SOCS3 interact with and modulate fibroblast growth factor receptor signaling. J Cell Sci. 2006;119:380-7.

49. Alexander WS, Hilton DJ. The role of suppressors of cytokine signaling (SOCS) proteins in regulation of the immune response. Annu Rev Immunol. 2004;22:503-29.

50. Karnaky KJ. Structure and function of the chloride cell of Fundulus heteroclitus and other teleosts. Integr Comp Biol. 1986;26:209-24.

51. Heisler N. In: Bridges CR, Butler PJ, editors. Techniques in comparative respiratory physiology: an environmental approach. Cambridge: Cambridge University Press; 1989. p. 305-32.

52. Claiborne JB, Edwards SL, Morrison-Shetlar Al. Acid-base regulation in fishes: cellular and molecular mechanisms. J Exp Zool. 2002;293:302-19.

53. Gilmour KM, Perry SF. Carbonic anhydrase and acid-base regulation in fish J Exp Biol. 2009;212:1647-61.

54. Evans DH, Piermarini PM, Choe KP. The multifunctional fish gill : dominant site of gas exchange, osmoregulation, acid-base regulation, and excretion of nitrogenous waste. 2005. p. 97-177. doi:10.1152/physrev.00050.2003.

55. Perry SF, Gilmour KM. Acid-base balance and CO2 excretion in fish: unanswered questions and emerging models. Respir Physiol Neurobiol. 2006;154:199-215

56. Bobulescu IA, Moe OW. Na+/H+ exchangers in renal regulation of acid-base balance. Semin Nephrol. 2006;26:334-44.

57. Miyazaki E, Sakaguchi M, Wakabayashi S, Shigekawa M, Mihara K. NHE6 protein possesses a signal peptide destined for endoplasmic reticulum membrane and localizes in secretory organelles of the cell. J Biol Chem. 2001;276:49221-7.

58. Lushchak VI. Environmentally induced oxidative stress in aquatic animals. Aquat Toxicol. 2011;101:13-30.

59. Lee $\mathrm{S}-\mathrm{O}$ et al. Protein disulphide isomerase is required for signal peptide peptidase-mediated protein degradation. EMBO J. 2010;29:363-75.

60. Kriegenburg F. Quality control of protein folding: an overview. FEBS J. 2012; 279:8457.

61. Hatahet F, Ruddock LW. Protein disulfide isomerase: a critical evaluation of its function in disulfide bond formation. Antioxid Redox Signal. 2009;11:2807-50.

62. Riedl SJ, Shi Y. Molecular mechanisms of caspase regulation during apoptosis. Nat Rev Mol Cell Biol. 2004;5:897-907.

63. Li H, Zhu H, Xu CJ, Yuan J. Cleavage of BID by caspase 8 mediates the mitochondrial damage in the Fas pathway of apoptosis. Cell. 1998;94:491-501.

64. Budihardjo I, Oliver H, Lutter M, Luo X, Wang X. Biochemical pathways of caspase activation during apoptosis. Annu Rev Cell Dev Biol. 1999;15:269-90.
65. Basler M, Kirk CJ, Groettrup M. The immunoproteasome in antigen processing and other immunological functions. Curr Opin Immunol. 2013;25:74-80.

66. Fangue NA et al. A laboratory-based, experimental system for the study of ocean acidification effects on marine invertebrate larvae. Limnol Oceanogr Methods. 2010;8:441-52.

67. Riebesell U, Fabry VJ, Hansson L. Gattuso J-P. (Eds.). Guide to best practices for ocean acidification research and data reporting, 260 p. Luxemborg: Publications Office of the European Union. 2010.

68. Robbins LL, Hansen ME, Kleypas JA, Meylan SC. CO2calc-a user-friendly seawater carbon calculator for Windows, Mac OS X and iOS (iPhone). U.S. Geol. Surv. Open-File Rep. 2010-1280. 2010. p. 17.

69. Mehrbach C, Culberson CH, Hawley JE, Pytkowicz RM. Measurement of the apparent dissociation constants of carbonic acid in seawater at atmospheric pressure. Limnol Oceanogr. 1973;18:897-907.

70. Dickson AG, Millero FJ. A comparison of the equilibrium constants for the dissociation of carbonic acid in seawater media. Deep Sea Res Part A Oceanogr Res Pap. 1987;34:1733-43.

71. Grabherr MG et al. Full-length transcriptome assembly from RNA-Seq data without a reference genome. Nat Biotechnol. 2011;29:644-52.

72. Pittsburgh Supercomputing Center-Blacklight Supercomputer. Blacklight Pittsburgh Supercomputing Center-Blacklight System Configuration. 2014. p. 1-14. at https://www.psc.edu/index.php/computing-resources/ blacklight\#arch.

73. Li B, Dewey CN. RSEM: accurate transcript quantification from RNA-Seq data with or without a reference genome. BMC Bioinformatics. 2011;12:323.

74. Fu L, Niu B, Zhu Z, Wu S, Li W. CD-HIT: accelerated for clustering the next-generation sequencing data. Bioinformatics. 2012;28:3150-2.

75. Texas Advanced Computing Center-Stampede Super Computer. System overview. 2014. p. 1-47. at https://www.tacc.utexas.edu/user-services/userguides/stampede-user-guide.

76. Camacho $\mathrm{C}$ et al. BLAST+: architecture and applications. BMC Bioinformatics. 2009;10:421.

77. BLAST2GO Command Line. Blast2GO Command Line (CLI) Please choose among the following options: Main Command Line Features. 2014. p. 1-2. at http://www.blast2go.com/blast2gocli.

78. Haas $B$ et al. De novo transcript sequence reconstruction from RNA-seq using the Trinity platform for reference generation and analysis. Nat Protoc. 2013;8(8):1494-412.

79. R Core Team. R: a language and environment for statistical computing. 2013. at URL http://www.R-project.org/.

80. Conesa A et al. Blast2GO: a universal tool for annotation, visualization and analysis in functional genomics research. Bioinformatics. 2005;21:3674-6.

\section{Submit your next manuscript to BioMed Central and we will help you at every step:}

- We accept pre-submission inquiries

- Our selector tool helps you to find the most relevant journal

- We provide round the clock customer support

- Convenient online submission

- Thorough peer review

- Inclusion in PubMed and all major indexing services

- Maximum visibility for your research

Submit your manuscript at www.biomedcentral.com/submit
Biomed Central 